Notfall Rettungsmed 2010 - 13:665-678

DOI 10.1007/s10049-010-1373-0

Online publiziert: 16.11 .2010

(c) European Resuscitation Council 2010

\author{
S. Richmond ${ }^{1}$. J. Wyllie ${ }^{2}$ \\ ${ }^{1}$ Sunderland Royal Hospital, Sunderland \\ ${ }^{2}$ The James Cook University Hospital, Middlesbrough
}

\title{
Versorgung und Reanimation des Neugeborenen
}

\section{Sektion 7 der Leitlinien zur Reanimation 2010 des European Resuscitation Council}

\section{Einführung}

Die folgenden Leitlinien zur Neugeborenenreanimation sind Ergebnis eines Prozesses, der mit der „International Consensus Conference on Emergency Cardiovascular Care (ECC) and Cardiopulmonary Resuscitation (CPR) Science with Treatment Recommendations 2010" seinen Abschluss fand $[1,2]$. Sie sind eine Erweiterung der bereits durch den European Resuscitation Council (ERC) veröffentlichten Leitlinien [3] und berücksichtigen zudem Empfehlungen nationaler sowie internationaler Organisationen.

\section{Zusammenfassung der Veränderungen der Leitlinien von 2005}

Für die Leitlinien 2010 ergeben sich folgende wesentliche Veränderungen (- Abb. 1):

- Für unbeeinträchtigte, gesunde Neugeborene wird ein verzögertes Abnabeln empfohlen. Das Abnabeln soll frühestens nach 1 min erfolgen. Für den optimalen Zeitpunkt des Abnabelns eines schwer beeinträchtigten Neugeborenen ist aufgrund fehlender Daten bisher keine Empfehlung möglich.

- Während der Reanimation eines reifen Neugeborenen soll Raumluft verwendet werden. Wenn trotz effektiver Ventilation die Oxygenierung (idea- lerweise durch Pulsoxymetrie überwacht) nicht zufriedenstellend ist, sollen höhere Sauerstoffkonzentrationen in Erwägung gezogen werden.

- Frühgeborene vor der 32. Schwangerschaftswoche (SSW) erreichen häufig unter Raumluft nicht die gleichen arteriellen Sauerstoffsättigungen wie reife Neugeborene. Für die Versorgung von Frühgeborenen soll daher eine durch Pulsoxymetrie überwachte, zielgerichtete Sauerstoffgabe mithilfe eines Sauerstoff-Raumluft-Mischers erfolgen. Steht kein Sauerstoff-Raumluft-Mischer zur Verfügung, muss verwendet werden, was verfügbar ist.

- Frühgeborene vor der 28. SSW sollen direkt nach der Geburt, ohne sie vorher abzutrocknen, vollständig bis zum Hals in durchsichtige Folie aus Plastik oder einen Plastiksack eingepackt werden. Die weitere Versorgung soll unter einem Wärmestrahler stattfinden. Das Frühgeborene soll in der Folie bis zur Kontrolle der Köpertemperatur nach der Aufnahme bleiben. Für Frühgeborene vor der 28. SSW soll die Raumtemperatur im Kreißsaal bei mindestens $26^{\circ} \mathrm{C}$ liegen.

- Das empfohlene Kompression-Ventilation- („compression-ventilation“-, CV-)Verhältnis bleibt für die kardiopulmonale Reanimation („cardiopulmonary resuscitation“, CPR) eines Neugeborenen bei 3:1.
- Das intrapartale Absaugen von Mekonium aus dem Nasen- und Rachenraum des Neugeborenen nach Geburt des Kopfes wird nicht empfohlen. Zeigt sich ein Neugeborenes bei mekoniumhaltigem Fruchtwasser nach der Geburt mit einem schlaffen Muskeltonus und apnoisch, sollen rasch der Oropharynx inspiziert und potenzielle Atemwegsverlegungen entfernt werden. Steht in der Intubation von Neugeborenen erfahrenes Personal zur Verfügung, kann es in dieser Situation sinnvoll sein, das Neugeborene zu intubieren und tracheal abzusaugen. Dauert der Intubationsversuch zu lange oder ist er nicht erfolgreich, soll mit einer Maskenbeatmung begonnen werden, insbesondere wenn das Neugeborene eine anhaltende Bradykardie zeigt.

- Für Adrenalin (Epinephrin) ist die i.v.Gabe mit einer Dosis von 10-30 $\mu \mathrm{g} / \mathrm{kg}$ KG empfohlen. Soll Adrenalin endotracheal verabreicht werden, ist wahrscheinlich eine Dosis von mindestens 50-100 $\mu \mathrm{g} / \mathrm{kgKG}$ notwendig, um einen ähnlichen Effekt wie bei der i.v.-Gabe von $10 \mu \mathrm{g} / \mathrm{kgKG}$ Adrenalin zu erzielen.

- Die Messung von Kohlendioxid $\left(\mathrm{CO}_{2}\right)$ in der Ausatemluft ist in Ergänzung zur klinischen Beurteilung die zuverlässigste Methode zum Nachweis einer

Beide Autoren haben zu gleichen Anteil zum Manuskript beigetragen. 


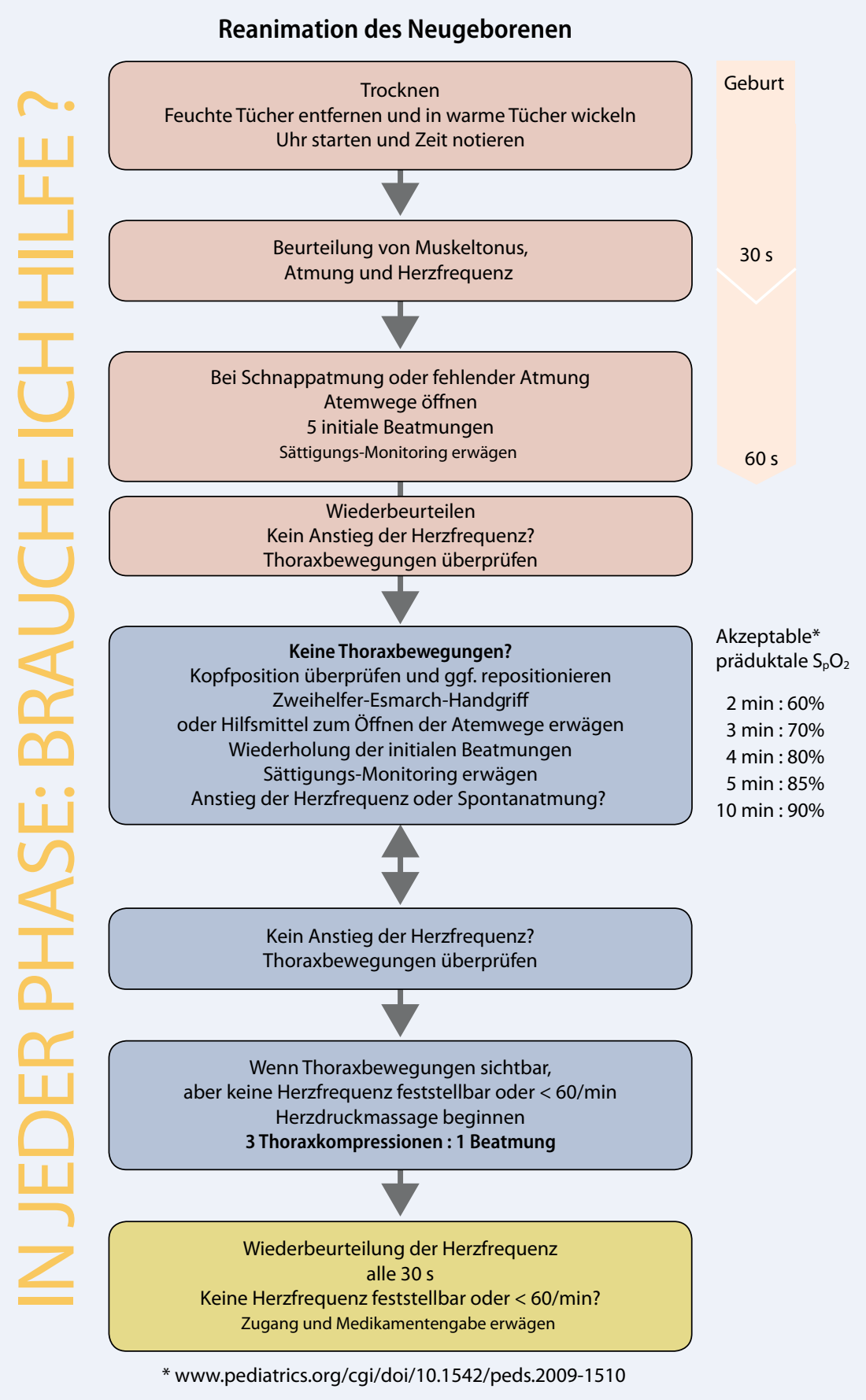

Abb. $1 \Delta$ Algorithmus der Neugeborenenreanimation

endotrachealen Tubuslage und wird bei Neugeborenen mit Spontankreislauf empfohlen.

- Bei reifen und nahezu reifen Neugeborenen mit moderater bis schwerer hypoxisch-ischämischer Enzephalopathie soll, wenn möglich, eine therapeutische Hypothermie durchgeführt werden. Dies betrifft nicht unmittelbar die Reanimationssituation, ist jedoch für die weiterführende Versorgung wichtig.
Die vorliegenden Leitlinien definieren nicht den einzig gangbaren Weg für die Reanimation eines Neugeborenen. Vielmehr entsprechen sie einer verbreiteten und akzeptierten Auffassung über die sichere sowie effektive Durchführung von Reanimationsmaßnahmen nach der Geburt.

\section{Vorbereitung}

Relativ wenige Neugeborene benötigen nach der Geburt Reanimationsmaß- nahmen. Sind Interventionen erforderlich, bestehen diese in der überwiegenden Zahl der Fälle lediglich in einer kurzen assistierten Belüftung der Lungen. Nur eine kleine Minderheit braucht, zusätzlich zur Lungenbelüftung, eine kurzzeitige Herzdruckmassage. Von 100.000 in einem Jahr in Schweden geborenen Babys mit einem Gewicht von über $2500 \mathrm{~g}$ waren bei lediglich 10 von 1000 (1\%) postnatal Reanimationsmaßnahmen notwendig [4]. Unter den reanimierten Neugeborenen war in 8 von 1000 Fällen eine Maskenbeatmung ausreichend, lediglich 2 von 1000 Neugeborenen mussten intubiert werden. Dieselbe Studie untersuchte die Häufigkeit unvorhergesehener Reanimationen nach der Geburt. Bei Neugeborenen mit geringem Risiko (problemlose Entbindung nach der 32. SSW) waren in lediglich 2 von 1000 Fällen $(0,2 \%)$ Reanimationsmaßnahmen notwendig. Von diesen sprachen $90 \%$ auf eine alleinige Maskenbeatmung an; die übrigen $10 \%$ besserten sich nicht durch die Maskenbeatmung und mussten intubiert werden.

Eine Reanimation des Neugeborenen oder die Notwendigkeit, einen Spezialisten zu einer Geburt hinzurufen zu müssen, ist wahrscheinlicher bei peripartalem Hinweis auf eine schwerwiegende Beeinträchtigung des Fetus, bei Frühgeborenen vor der 35. SSW, Neugeborenen nach Zangengeburt oder Mehrlingen. Da es häufig, aber eben nicht immer möglich ist, die Notwendigkeit von Reanimationsmaßnahmen vorherzusehen, soll bei jeder Geburt in der Neugeborenenreanimation ausgebildetes Personal leicht verfügbar sein. Ist eine Reanimation notwendig, kümmert sich dieses ausschließlich um die Versorgung des Neugeborenen. Ein in der endotrachealen Intubation eines Neugeborenen Erfahrener soll bei Hochrisikoentbindungen mit erhöhter Reanimationswahrscheinlichkeit bereits während der Geburt anwesend sein. Basierend auf aktueller Praxis und klinischer Qualitätsprüfung sollen lokale Leitlinien entwickelt werden, die festlegen, welches Personal bei jeder Geburt anwesend sein soll.

Ein strukturiertes Ausbildungsprogramm für Standards und Fertigkeiten der Neugeborenenreanimation ist daher für jede geburtshilfliche Einrichtung unabdingbar. 


\section{Geplante Hausgeburten}

Empfehlungen, welche Personen bei einer geplanten Hausgeburt anwesend sein sollen, sind länderspezifisch unterschiedlich. Ist die Entscheidung für eine Hausgeburt in Abstimmung mit dem Arzt und der Hebamme gefallen, gelten auch für die Hausgeburt die Standards der Neugeborenenversorgung. Bereits bei der Planung einer Hausgeburt muss der Mutter deutlich gemacht werden, dass aufgrund der schwierigeren Verfügbarkeit weiterer Hilfe eine Reanimation in häuslicher Umgebung nicht im vollen Umfang durchgeführt werden kann. Idealerweise sollen bei allen Hausgeburten 2 trainierte professionelle Helfer anwesend sein; mindestens einer der beiden muss in der Durchführung von Maskenbeatmung und Herzdruckmassagen bei Neugeborenen gut ausgebildet und erfahren sein.

\section{Ausrüstung und Vorbereitungen}

Im Gegensatz zu Reanimationen beim Erwachsenen, ist eine Reanimation nach der Geburt oft ein vorhersehbares Ereignis. Daher ist es meist möglich, Umgebung und Ausrüstung noch vor der Entbindung eines Babys vorzubereiten. Die Versorgung eines kritisch kranken Neugeborenen soll möglichst in einer warmen, gut beleuchteten und zugluftfreien Umgebung stattfinden. Das Baby soll unter einen Heizstrahler auf eine gerade und flache Fläche gelegt werden. Sämtliches, zur Reanimation notwendiges Material muss leicht verfügbar sein. Es muss regelmäßig auf Vollständigkeit und Funktion überprüft werden.

Findet eine Geburt außerhalb der üblichen Entbindungsbereiche statt, soll als Mindestausstattung nachfolgende Ausrüstung zur Verfügung stehen:

- ein Hilfsmittel zur sicheren, assistierten Beatmung (Beatmungsbeutel/T-

Stück) und Maske in passender Größe für Neugeborene,

- warme, trockene Tücher und Laken,

- ein steriles Instrument zum Durchtrennen der Nabelschnur und

- saubere Handschuhe für die Versorgenden.

- Hilfreich wären außerdem eine Absaugeinheit mit passenden Absaug- kathetern sowie ein Spatel (bzw. ein Laryngoskop) zur Einsicht in den Oropharynx.

Da unerwartete Geburten außerhalb des klinischen Umfelds v. a. das Personals des Rettungsdienstes betreffen, sollen diese auf die Versorgung von Neugeborenen vorbereitet sein.

\section{Wärmemanagement}

Nackte, feuchte Neugeborene sind in einem Raum, der für Erwachsene angenehm warm erscheint, nicht in der Lage, ihre Körpertemperatur zu halten. Beeinträchtigte Neugeborene sind diesbezüglich besonders empfindlich [5]. Wird das Neugeborene einem Kältestress ausgesetzt, führt dies zu einer verminderten arteriellen Sauerstoffkonzentration [6] und einer zunehmenden metabolischen Acidose [7].

Einem Wärmeverlust soll folgendermaßen vorgebeugt werden:

- Das Neugeborene vor Zugluft schützen.

- Den Entbindungsraum warm halten. Für Frühgeborene vor der 28. SSW soll die Raumtemperatur bei $26^{\circ} \mathrm{C}$ liegen $[8,9]$.

- Das reife Neugeborene direkt nach der Geburt sorgfältig abtrocknen. Um weiteren Wärmeverlust zu vermeiden, den Kopf und Körper des Neugeborenen, unter Aussparung des Gesichts, mit einem warmen Tuch bedecken.

Alternativ kann das Neugeborene der Mutter Haut auf Haut auf den Bauch gelegt und beide können mit einem Tuch zugedeckt werden.

- Sind Reanimationsmaßnahmen notwendig, wird das Baby unter einem vorgewärmten Heizstrahler auf einer warmen, ebenen Fläche platziert.

- Bei sehr unreifen Frühgeborenen (insbesondere vor der 28. SSW) kann es sein, dass Trocknen und Einwickeln in warme Tücher nicht ausreichen. Ein effektiveres Wärmemanagement lässt sich für diese Kinder erzielen, wenn der Körper und der Kopf des Kindes, unter Aussparen des Gesichts, in eine durchsichtige Plastikfolie gehüllt werden. Das Kind wird vorher nicht abgetrocknet und so eingehüllt unter einem Wärmestrahler platziert.

\section{Initiale Beurteilung}

Der Apgar-Scorewarals, ,einfache, gebräuchliche, eindeutige Klassifikation des Neugeborenen nach der Geburt" gedacht, und soll „als Diskussionsgrundlage und zum Vergleich verschiedener geburtshilflicher Praktiken, Verfahren zur mütterlichen Schmerztherapie und zur Beurteilung von Reanimationsmaßnahmen dienen (Betonung durch die Autoren; [10]). Er ist nicht zur Identifikation von Neugeborenen, die Reanimationsmaßnahmen benötigen, entwickelt worden und dafür auch nicht geeignet.

Allerdings können einige Parameter des Scores, wie Atmung, Herzfrequenz und Muskeltonus, wenn sie rasch und simultan erfasst werden, Neugeborene identifizieren, die einer Reanimation bedürfen. (Virginia Apgar selbst beschrieb die Herzfrequenz als aussagekräftigsten Parameter für die rasche Erholung eines beeinträchtigten Neugeborenen [10].) Die wiederholte Erhebung der Herzfrequenz, mehr als die Beurteilung der Atmung, ist ein guter Parameter, um zu beurteilen, ob sich der Zustand eines Neugeborenen unter den durchgeführten Maßnahmen bessert oder weitere Maßnahmen notwendig sind.

\section{Atmung}

Überprüfen Sie, ob das Neugeborene atmet. Wenn es atmet, beurteilen Sie die Atemfrequenz sowie die Tiefe und die Symmetrie der Atemexkursionen. Achten Sie auf Zeichen pathologischer Atemmuster, wie Schnappatmung oder stöhnen (knorksen).

\section{Herzfrequenz}

Die beste Methode zur Beurteilung der Herzfrequenz ist die direkte Auskultation mit dem Stethoskop über der Herzspitze. Das Tasten des Pulses an der Basis der Nabelschnur ist oft möglich, kann aber irreführend sein. Eine Beurteilung der Herzfrequenz allein über die Pulsation der Nabelschnur ist nur zuverlässig, wenn die Herzfrequenz über 100 Schlägen/min liegt. Für Neugeborene, die eine Reanimation und/oder Unterstützung bei der Atmung brauchen, kann ein modernes Pulsoxymeter zur Bestimmung der Herzfrequenz hilfreich sein [13]. 


\section{Hautkolorit}

Das Hautkolorit ist ein schlechter Parameter, um die Oxygenierung zu beurteilen. Diese soll, wenn möglich, mithilfe der Pulsoxymetrie erfasst werden. Ein gesundes Neugeborenes ist unmittelbar nach der Geburt zunächst zyanotisch und wird bei effektiver Spontanatmung innerhalb von 30 s rosig. Eine periphere Zyanose ist häufig und in den wenigsten Fällen Zeichen einer Hypoxie. Eine ausgeprägte, persistierende Blässe trotz effektiver Ventilationen kann Zeichen einer signifikanten Acidose, seltener einer Hypovolämie sein. Auch wenn die visuelle Beurteilung des Hautkolorits eine schlechte Methode zur Erfassung einer Zyanose ist, soll sie nicht unterbewertet werden: Überprüfen Sie die Oxygenierung des Neugeborenen unbedingt mithilfe der Pulsoxymetrie, wenn Ihnen ein Neugeborenes zyanotisch erscheint.

\section{Muskeltonus}

Ein deutlich hypotones Neugeborenes ist zumeist auch bewusstlos und benötigt respiratorische Unterstützung.

\section{Taktile Stimulation}

Das Abtrocknen des Neugeborenen bedeutet i. Allg. eine ausreichende Stimulation zur Anregung einer effektiven Spontanatmung. Eine übertrieben kräftige Stimulation soll vermieden werden. Entwickelt das Baby trotz taktiler Stimulation keine effektive Spontanatmung, sind weitere unterstützende Maßnahmen notwendig.

\section{Einteilung nach der initialen klinischen Beurteilung}

Anhand der initialen klinischen Beurteilung lassen sich Neugeborene in 3 Gruppen einteilen:

\section{Kräftiges Schreien/suffiziente At- mung, \\ - guter Muskeltonus, \\ - Herzfrequenz $>100 / \mathrm{min}$.}

Neugeborene dieser Gruppe benötigen keine weiteren Maßnahmen außer Abtrocknen und Einwickeln in warme Tücher. Das Neugeborene kann der Mutter auf den Bauch gelegt werden. Durch den Kontakt zur Haut der zugedeckten Mutter wird das Baby gewärmt. Es kann zu diesem Zeitpunkt bereits an die Brust angelegt werden.

2. Insuffiziente Spontanatmung oder Apnoe,

- normaler bis reduzierter Muskeltonus, - Herzfrequenz $<100 / \mathrm{min}$.

Nachdem ein Neugeborenes dieser Gruppe getrocknet und in warme Tücher gewickelt wurde, ist meist eine kurze Maskenbeatmung ausreichend. Kommt es darunter nicht zu einem adäquaten Anstieg der Herzfrequenz, müssen Herzdruckmassagen durchgeführt werden.

3. Insuffiziente Spontanatmung oder Apnoe,

- schlaffer Muskeltonus („floppy“),

- Bradykarde oder nichtnachweisbare Herzfrequenz,

- oft mit ausgeprägter Blässe als Zeichen einer schlechten Perfusion.

Nachdem ein Neugeborenes dieser Gruppe getrocknet und in warme Tücher gewickelt wurde, müssen unverzüglich die Atemwege geöffnet werden. Die Lungen müssen belüftet werden, und das Kind muss beatmet werden. Wurden die Atemwege erfolgreich geöffnet und die Lungen belüftet, können außerdem Herzdruckmassagen und möglicherweise eine Medikamentengabe notwendig sein.

Eine sehr kleine Gruppe von Neugeborenen bleibt trotz adäquater Spontanatmung und guter Herzfrequenz hypoxämisch. Die Diagnosen umfassen hier ein weites Spektrum, wie z. B. eine Zwerchfellhernie, Surfactant-Mangel, eine kongenitale Pneumonie, einen Pneumothorax oder ein angeborenes zyanotisches Herzvitium.

\section{Reanimation des Neugeborenen}

Wenn Sie bei der initialen Beurteilung feststellen, dass das Neugeborene keine suffiziente und regelmäßige Spontanatmung entwickelt oder eine Herzfrequenz $<100 /$ min hat, muss mit Reanimationsmaßnahmen begonnen werden.
Meist sind lediglich ein Freimachen der Atemwege und die Belüftung der Lungen notwendig. Bedenken Sie allerdings, dass alle weiteren Maßnahmen erfolglos bleiben werden, wenn diese ersten beiden Schritte, das Öffnen der Atemwege und die Belüftung der Lungen, nicht effektiv durchgeführt wurden.

\section{Atemwege}

Lagern Sie das Neugeborene in Rückenlage mit dem Kopf in Neutralposition (- Abb. 2). Zur optimalen Lagerung und Stabilisierung des Kopfes in Neutralposition kann die Platzierung eines $2 \mathrm{~cm}$ dicken Lakens oder Tuchs unter den Schultern des Neugeborenen hilfreich sein. Um die Atemwege eines Neugeborenen mit schlaffem Muskeltonus zu öffnen, kann der Esmarch-Handgriff oder die Verwendung eines oropharyngealen Tubus (Guedel-Tubus; Anmerkung der Übersetzer) in passender Größe nützlich sein.

Absaugen ist nur notwendig, wenn die Atemwege verlegt sind. Eine solche Verlegung kann aufgrund von Mekonium, auch wenn das Neugeborene keine Mekoniumablagerungen auf der Haut zeigt, aber auch Blutkoageln, zähem Schleim oder Vernix bestehen. Wird abgesaugt, ist zu bedenken, dass zu heftiges oropharyngeales Absaugen das Einsetzen einer suffizienten Spontanatmung verzögern und zu einem Laryngospasmus sowie zu einer vagalen Bradykardie führen kann [15]. Das Vorhandensein von zähflüssigem Mekonium beim schlaffen, avitalen Neugeborenen ist die einzige Situation, in der ein sofortiges Absaugen des Oropharynx $z$ u erwägen ist. Wenn ein Absaugen des Oropharynx erwogen wird, soll dies idealerweise unter Sicht erfolgen. Verwenden Sie einen 12- bis 14-F-Absaugkatheter oder einen Yankauer-Absauger. Der applizierte Sog soll nicht mehr als $-100 \mathrm{mmHg}$ betragen.

\section{Atmung}

Nachdem das Neugeborene getrocknet, in Tücher eingewickelt wurde und die Atemwege geöffnet wurden, hat bei fehlender oder insuffizienter Spontanatmung, die Belüftung der Lungen die oberste Priorität (- Abb. 3). Beginnen Sie bei reifen Neugeborenen die Beatmung mit Raumluft. 
Das wichtigste Kriterium zur Beurteilung einer adäquaten Lungenentfaltung und belüftung ist eine rasche Verbesserung der Herzfrequenz. Kommt es zu keinem Anstieg der Herzfrequenz, muss überprüft werden, ob sich der Thorax adäquat hebt und senkt.

Halten Sie für die ersten 5 Beatmungen den Inspirationsdruck über jeweils 2-3 s aufrecht. Dies erleichtert die Entfaltung der Lungen. Die meisten Neugeborenen, die Reanimationsmaßnahmen benötigen, zeigen innerhalb von $30 \mathrm{~s}$ unter Ventilation der Lungen einen raschen Anstieg der Herzfrequenz. Steigt die Herzfrequenz an, aber das Neugeborene weist keine suffiziente Spontanatmung auf, wird mit einer Frequenz von 30 Beatmungen/min weiterbeatmet, bis eine suffiziente Spontanatmung einsetzt. Die Inspirationszeit soll nun während der Beatmung bei $1 \mathrm{~s}$ liegen. Ist die passive Beatmung suffizient, ist dies in der Regel an einem sofortigen Anstieg der Herzfrequenz bzw. wenn sich die Herzfrequenz über 100 Schlägen/min hält, erkennbar. Reagiert das Neugeborene nicht entsprechend, liegt die Ursache in den meisten Fällen in einer insuffizienten Öffnung der Atemwege oder einer nichteffektiven Beatmung. Achten Sie bei der Beatmung auf passive Thoraxbewegungen, die eine adäquate Entfaltung der Lungen anzeigen. Hebt sich der Thorax nicht, ist der Atemweg nicht unter Kontrolle, und die Lungen sind nicht suffizient belüftet. Ohne suffiziente Belüftung der Lungen können Herzdruckmassagen nicht wirksam sein. Bevor mit Herzdruckmassagen begonnen wird, muss unbedingt die effektive Ventilation der Lungen sichergestellt sein.

Eine Möglichkeit der Sicherung der Atemwege ist die endotracheale Intubation. Diese benötigt jedoch Training und Erfahrung. Ist niemand anwesend, der eine Intubation bei einem Neugeborenen durchführen kann, und es kommt zu keiner Stabilisierung der Herzfrequenz, muss erneut die Kopfposition überprüft und Beatmungen mit verlängerter Inspirationszeit müssen durchgeführt werden. Währenddessen soll ein Helfer mit ausreichender Intubationserfahrung zur Hilfe gerufen werden.

Fahren Sie mit der Beatmung fort, bis das Neugeborene eine suffiziente, regelmäßige Spontanatmung zeigt.

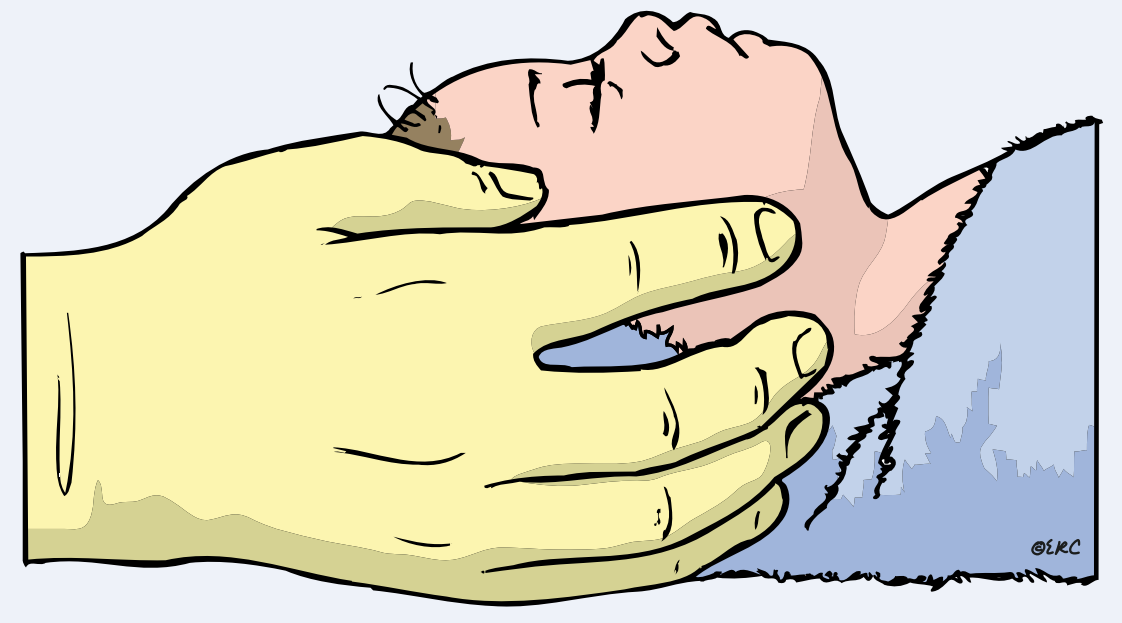

Abb. $2 \Delta$ Neutralposition des Kopfes (beim Neugeborenen)

Abb. 3 Maskenventilation (des Neugeborenen)

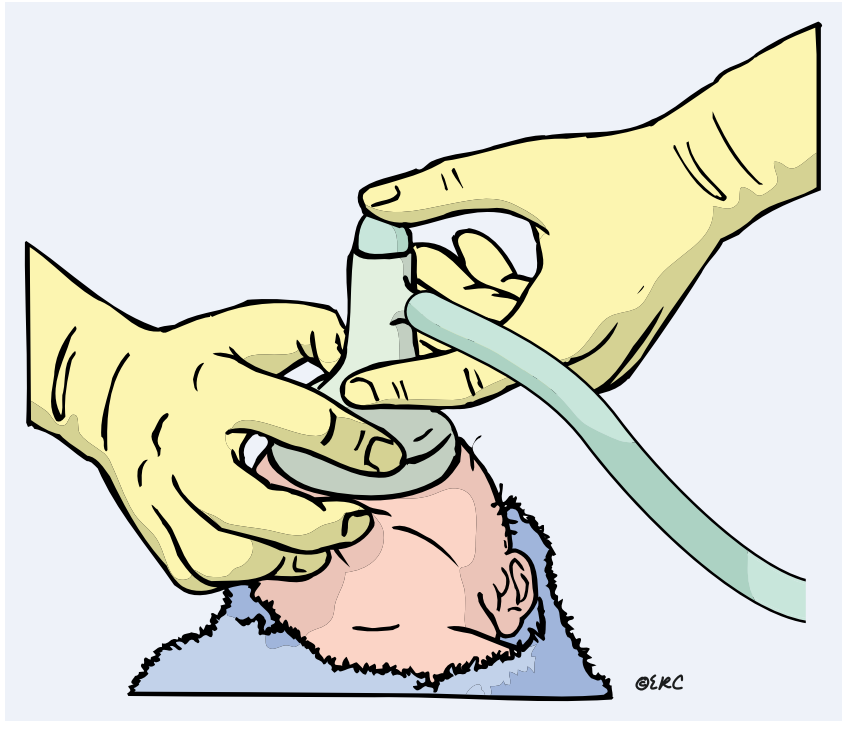

\section{Herz-Kreislauf-Unterstützung}

Eine Unterstützung von Herz und Kreislauf durch Herzdruckmassagen kann nur wirksam sein, wenn die Lungen zuvor erfolgreich belüftet wurden. Beginnen Sie mit den Thoraxkompressionen, wenn die Herzfrequenz trotz adäquater Ventilation unter 60 Schlägen/min liegt.

Platzieren Sie für eine optimale Technik 2 Daumen nebeneinander über dem unteren Drittel des Brustbeins, direkt unter einer gedachten Linie zwischen den Brustwarzen. Umgreifen Sie mit den Fingern den gesamten Brustkorb und unterstützen Sie so den Rücken des Kindes (- Abb. 4, [16, 17, 18, 19]).

Zum Auffinden des korrekten Druckpunkts kann alternativ die Spitze des Brustbeins aufgesucht und die Daumen können einen Finger breit darüber platziert wer- den. Das Brustbein soll um ein Drittel des anterioposterioren Thoraxdurchmessers komprimiert werden und muss nach jeder Kompression wieder in die Ausgangsposition zurückkommen [20].

Führen Sie Thoraxkompressionen und Beatmungen in einem Verhältnis von 3:1 durch. Dies bedeutet etwa 120 Maßnah$\mathrm{men} / \mathrm{min}$. So erreichen Sie etwa 90 Kompressionen und 30 Beatmungen $/ \mathrm{min}$. Eine gegenüber der Kompressionsphase gering verlängerte Relaxationsphase bietet theoretische Vorteile [21]. Letztlich ist die Qualität der Thoraxkompressionen und der Beatmung wahrscheinlich entscheidender als deren Frequenz.

Überprüfen Sie alle 30 s die Herzfrequenz, und beenden Sie die Thoraxkompressionen erst, wenn die Herzfrequenz über $60 / \mathrm{min}$ liegt. 


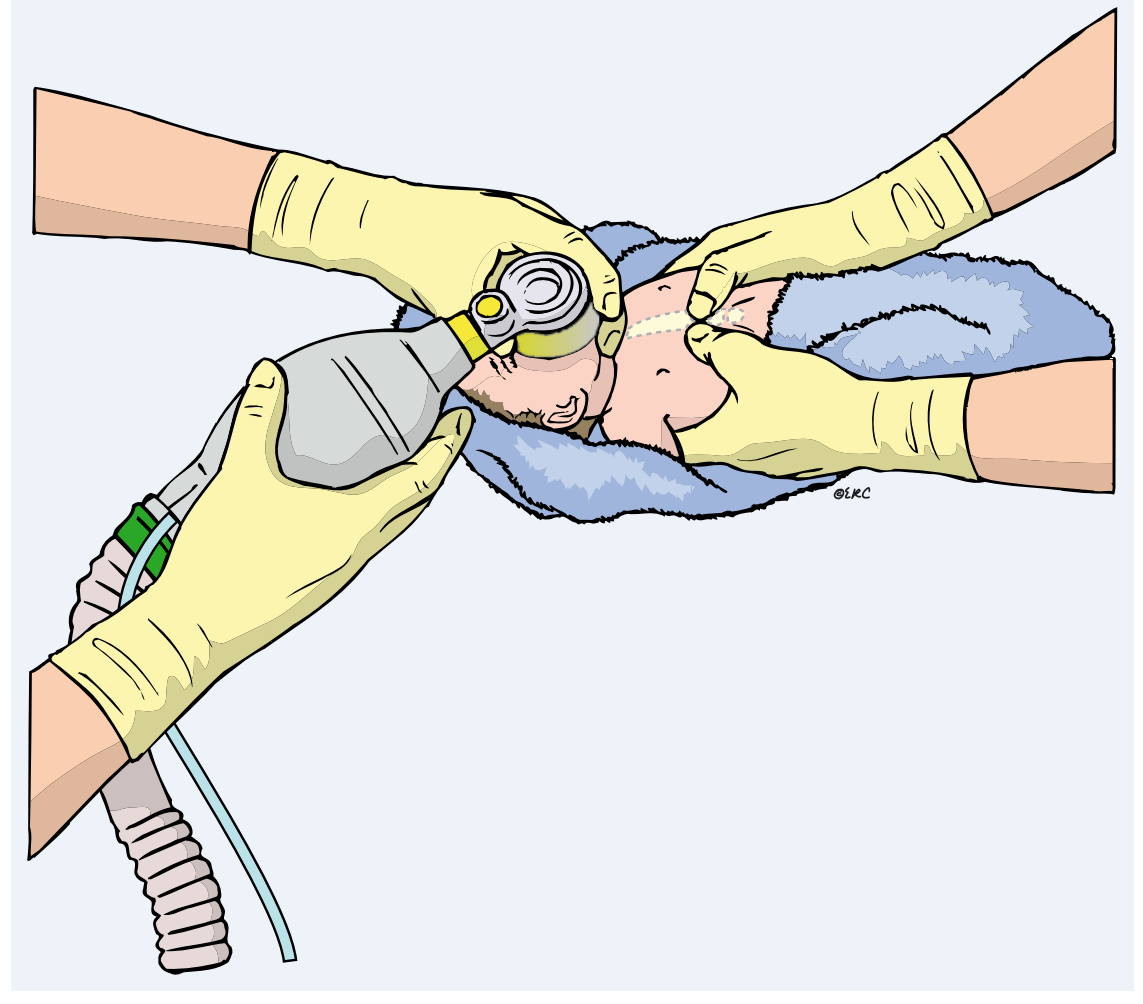

Abb. $4 \Delta$ Beatmungen und Thoraxkompressionen (beim Neugeborenen)

\section{Medikamente}

Die Gabe von Medikamenten ist während der Reanimation eines Neugeborenen selten erforderlich. Eine Bradykardie ist beim Neugeborenen in den meisten Fällen entweder durch eine inadäquate Ventilation oder eine schwere Hypoxie verursacht. Eine adäquate Beatmung ist daher die wichtigste Maßnahme zur Behebung der Bradykardie. Bleibt die Herzfrequenz trotz suffizienter Beatmung und Thoraxkompressionen unter $60 / \mathrm{min}$, soll die Gabe von Medikamenten erwogen werden. Die Gabe erfolgt idealerweise über einen Nabelvenenkatheter (• Abb.5).

\section{Adrenalin}

Trotz unzureichender Daten aus Studien am Menschen scheint die Verwendung von Adrenalin sinnvoll, wenn die Herzfrequenz, trotz adäquater Beatmung und suffizienter Herzdruckmassagen, nicht über 6o/min ansteigt. Die empfohlene i.v.-Dosis beträgt $10-30 \mu \mathrm{g} / \mathrm{kgKG}$ und soll sobald wie möglich verabreicht werden.

Eine endotracheale Gabe wird nicht empfohlen (s. unten). Wird Adrenalin dennoch endotracheal verabreicht, sind sehr wahrscheinlich Dosen von 50-
$100 \mu \mathrm{g} / \mathrm{kgKG}$ notwendig. Allerdings wurde bisher weder die Sicherheit noch die Effektivität der höheren endotrachealen Dosis untersucht. Die hohe Dosis soll nicht i.v. gegeben werden.

\section{Natriumbikarbonat}

Kommt es unter suffizienter Beatmung und effektiven Thoraxkompressionen nicht zur Herstellung eines Spontankreislaufs, lässt sich die Myokardfunktion möglicherweise durch die Korrektur einer bestehenden myokardialen Acidose verbessern und ein Spontankreislauf herstellen. Um eine Routinegabe von Natriumbikarbonat während der Reanimation eines Neugeborenen zu empfehlen, fehlen die entsprechenden Daten. Natriumbikarbonat ist hyperosmolar und führt bei Gabe zu einer von Kohlendioxidfreisetzung. Dies kann die myokardiale und die zerebrale Funktion beeinträchtigen. Während einer kurzen Reanimationsphase ist die Gabe von Natriumbikarbonat daher nicht empfohlen. Im Rahmen eines prolongierten Atem-Kreislauf-Stillstands soll die Gabe von Natriumbikarbonat erst nach Herstellung einer effektiven Ventilation und nach Durchführung effektiver Thoraxkompressionen erwogen werden. Natri- umbikarbonat soll nach Herstellung effektiver Ventilationen und Thoraxkompressionen langsam i.v. in einer Dosis von 1-2 mmol/kgKG verabreicht werden.

\section{Flüssigkeitsgabe}

Erwägen Sie eine Gabe von Flüssigkeit, wenn der Verdacht auf einen Blutverlust besteht oder das Neugeborene Zeichen eines Schocks aufweist (Blässe, schlechte periphere Durchblutung, schwache Pulse) und auf adäquate Reanimationsmaßnahmen nicht anspricht [22]. Dies ist allerdings sehr selten der Fall. Ist im Fall eines Blutverlusts kein geeignetes Blut verfügbar (d. h. bestrahltes, leukozytendepletiertes, o-Rhnegatives Erythrozytenkonzentrat), ist zur Erhöhung des intravasalen Volumens isotonisch-kristalloiden Lösungen vor albuminhaltigen Lösungen der Vorzug zu geben.

Geben Sie einen Flüssigkeitsbolus von $10 \mathrm{ml} / \mathrm{kgKG}$. Kommt es darunter zu einer klinischen Verbesserung, können im Verlauf ggf. wiederholte Bolusgaben notwendig sein.

\section{Beendigung von \\ Reanimationsmaßnahmen}

Regionale und nationale Gremien legen in den meisten Ländern Richtlinien zur Beendigung von Reanimationsmaßnahmen fest. Zeigt ein gerade geborenes Kind keine Herzfrequenz und bleibt die Herzfrequenz für weitere 10 min aus, kann die Beendigung von Reanimationsmaßnahmen in Erwägung gezogen werden.

Die Entscheidung, ob eine Reanimation nach 10 min ohne erkennbare Herzfrequenz beendet wird, ist oft komplex und wird durch die weiteren Umstände bestimmt. Es fließt eine Vielzahl von Variablen ein, wie die Ätiologie des AtemKreislauf-Stillstands, das Gestationsalter, die Aussicht auf Reversibilität des Zustands und zuvor von den Eltern geäußerte Ansichten über ein vertretbares Risiko einer Morbidität des Kindes.

Liegt die Herzfrequenz nach der Geburt unter $60 / \mathrm{min}$, und es kommt trotz adäquater Reanimationsmaßnahmen nach 10-15 min nicht zu einem signifikanten Anstieg der Herzfrequenz, ist die Entscheidung zur Beendigung von Reanimationsmaßnahmen deutlich schwieriger. 
Für diese Situation gibt es keine evidenten Daten über die Prognose eines Kindes, um klare Empfehlungen für ein Weiterführen oder einen Abbruch der Reanimationsmaßnahmen zu geben.

\section{Kommunikation mit den Eltern}

Das erstversorgende Team soll die Eltern unbedingt über den Zustand des Kindes im Verlauf unterrichten. Halten Sie sich unter der Geburt an die lokale Routine und übergeben Sie das Neugeborene so frühzeitig wie möglich der Mutter. Sind Reanimationsmaßnahmen notwendig, sollen die Eltern über die durchgeführten Maßnahmen und deren Gründe aufgeklärt werden.

In die Entscheidung, ob und wann eine Neugeborenenreanimation beendet wird, soll idealerweise ein erfahrener Kinderarzt eingebunden sein. Ob Reanimationsmaßnahmen bei extremer Frühgeburtlichkeit eingeleitet werden, soll - wenn irgend möglich - in enger Abstimmung mit den Eltern und mit erfahrenen Kinderärzten sowie Geburtshelfern entschieden werden. Sind bei einem Kind bereits pränatal z. B. schwere, angeborene Fehlbildungen bekannt, sollen die entsprechenden Therapiemöglichkeiten und die weitere Prognose bereits vor der Geburt mit Eltern, Hebammen, Geburtshelfern und erstversorgendem Team besprochen werden.

Alle Gespräche und Entscheidungen müssen vor der Geburt sorgfältig im Krankenblatt der Schwangeren und postnatal im Krankenblatt des Neugeborenen dokumentiert werden.

\section{Spezielle Fragestellungen der „2010 Consensus Conference on CPR Science"}

\section{Aufrechterhaltung der Körpertemperatur bei Frühgeborenen}

Trotz Durchführung der üblicherweise empfohlenen Methoden gegen Wärmeverlust (Trocknen, Wickeln in warme Tücher und Platzierung unter einem Wärmestrahler) kühlen sehr kleine Frühgeborene häufig erheblich aus [24]. In mehreren randomisierten kontrollierten Stu-

1 Nabelvene

(ohne Schnur)

2 Nabelarterien

(ohne Schnur)

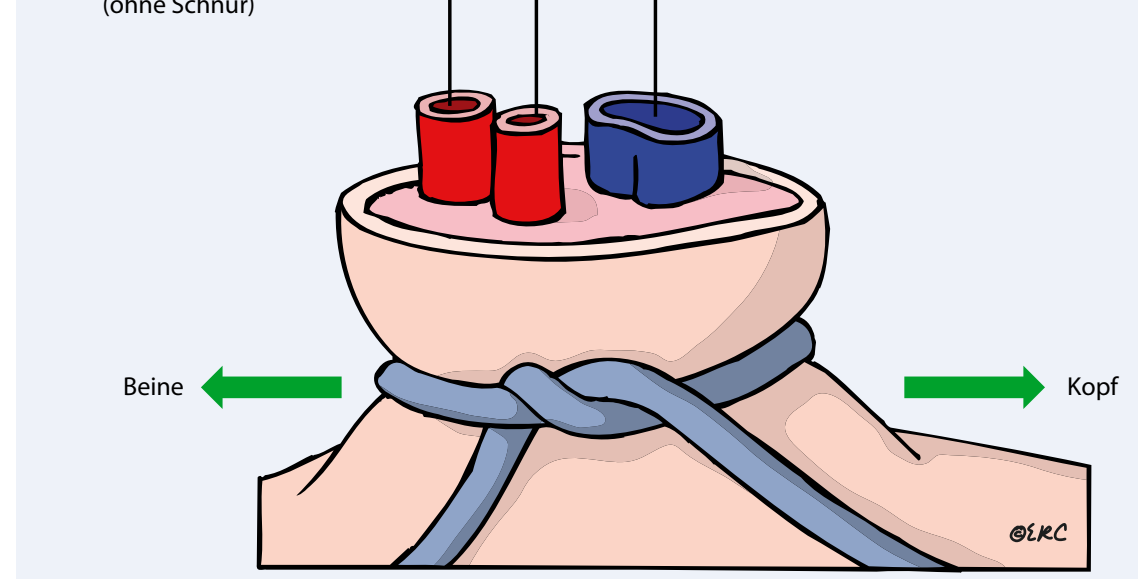

Abb. $5 \Delta$ Nabelschnur mit 2 Arterien und 1 Vene

dien und in Beobachtungsstudien konnte nachgewiesen werden, dass bei Lagerung des Frühgeborenen unter einem Heizstrahler und anschließendem Einwickeln in eine durchsichtige Plastikfolie ohne vorheriges Abtrocknen die Körpertemperatur bei Aufnahme auf der Intensivstation im Vergleich zu den herkömmlichen Methoden des Wärmemanagements deutlich höher ist $[25,26,27]$.

Aufgrund des geringen, jedoch beschriebenen Risikos einer Hyperthermie muss bei dieser Methode die Körpertemperatur engmaschig überwacht werden [28]. Alle notwendigen Reanimationsmaßnahmen wie Intubation, Herzdruckmassagen und das Legen von Zugängen können durchgeführt werden, während sich das Frühgeborene in der Folie befindet. Sehr kleine Frühgeborene sind besser in der Lage, ihre Temperatur zu halten, wenn die Umgebungstemperatur im Kreißsaal bei $26^{\circ} \mathrm{C}$ oder höher liegt $[8,9]$.

Neugeborene febriler Mütter zeigen eine höhere Inzidenz für eine postnatale Anpassungsstörung, neonatale Krampfanfälle, Frühmortalität und Zerebralparese [28, 29, 30]. Daten aus Tierversuchen weisen darauf hin, dass eine Hyperthermie während oder nach einer zerebralen Ischämie mit einer Zunahme der zerebralen Schädigung verbunden ist $[31,32]$. Eine Hyperthermie soll daher vermieden werden.

\section{Mekonium}

In der Vergangenheit bestand die Vorstellung, dass die Entfernung von Mekonium aus den Atemwegen eines Neugeborenen direkt nach der Geburt die Häufigkeit und die Schwere eines Mekoniumaspirationssyndroms (MAS) vermindern würde. Die Studien, die diese Vorgehensweise unterstützten, verglichen ihre Ergebnisse bezüglich des Outcome nach Absaugen von Mekonium mit dem Outcome historischer Kontrollgruppen [33, 34]. Weitere Studien konnten den Vorteil dieser Vorgehensweise nicht nachweisen $[35,36]$. Eine im Jahr 2000 publizierte, randomisierte kontrollierte Multizenterstudie zeigte, dass die elektive Intubation und das Absaugen von aspiriertem Mekonium aus der Trachea bei vitalen Neugeborenen das Auftreten eines MAS nicht verringern. Eine randomisierte Multizenterstudie aus dem Jahr 2004 demonstrierte zudem, dass das Absaugen von Mekonium aus Nase und Mund nach Entwicklung des Kopfs, aber vor Geburt der Schultern (intrapartales Absaugen), ebenso nicht effektiv ist [38]. Das intrapartale Absaugen und die elektive Intubation sowie das endotracheale Absaugen eines vitalen Kindes auch bei Vorliegen von mekoniumhaltigem Fruchtwasser sind daher nicht empfohlen.

Weiter unklar bleibt, wie bei mekoniumhaltigen Fruchtwasser vorgegangen werden soll, wenn das Neugeborene nicht vital ist. Beobachtungsstudien haben belegt, dass diese Kinder ein erhöhtes Risiko haben, ein MAS zu entwickeln. Allerdings gibt es bisher keine randomisierten Studien, die den Effekt einer Intubation, gefolgt von endotrachealem Absaugen gegenüber keiner Intubation untersucht haben. 
Empfehlung. Aufgrund fehlender randomisierter kontrollierter Studien gibt es derzeit keine Evidenz, die bisherige Praxis, ein avitales Neugeborenes mit mekoniumhaltigem Fruchtwasser, wenn möglich, unter Sicht oropharyngeal und endotracheal abzusaugen, zu ändern. Soll der Intubationsversuch zu lange dauern oder nicht erfolgreich sein, soll mit einer Maskenbeatmung begonnen werden. Dies gilt insbesondere, wenn das Kind eine anhaltende Bradykardie zeigt.

\section{Raumluft oder 100\%iger Sauerstoff}

Der Schlüssel zur erfolgreichen Reanimation eines Neugeborenen nach der Geburt ist die schnelle Etablierung eines pulmonalen Gasaustausches nach Ende der Sauerstoffversorgung über die Plazenta. In der Vergangenheit erschien es, dass die durch anaerobe Prozesse ausgelöste Zellschädigung durch die Bereitstellung möglichst hoher Sauerstoffkonzentrationen an von Hypoxie bedrohtes Gewebe vermindert werden könnte. In den letzten 30 Jahren gewann der Begriff des „Sauerstoffparadoxons" zunehmend an Bedeutung. Dieses besagt, dass die Zell- und Gewebeschädigung zunimmt, wenn ein zuvor hypoxisches Gewebe hohen Konzentrationen von Sauerstoff ausgesetzt wird. Die Rollen freier Radikale und Antioxidanzien im Rahmen der Apoptose und des Reperfusionsschadens wurden zunehmend erforscht und das Modell des oxidativen Stresses entwickelt. Die Unbedenklichkeit der Applikation von hohen Sauerstoffkonzentrationen, für wie kurz auch immer, ließ sich im Licht dieser Erkenntnisse zunehmend schwerer rechtfertigen. Gleichzeitig erbrachten randomisierte Studien an asphyktischen Neugeborenen deutliche Hinweise, dass Raumluft genauso effektiv, wenn nicht effektiver ist als 100\%iger Sauerstoff, zumindest bezüglich des kurzfristigen Outcome [39].

In Tierversuchen und Humanstudien konnte nachgewiesen werden, dass eine Hyperoxie allein, besonders nach einer Asphyxie, zu Schädigungen im Gehirn und in anderen Organen auf zellulärer Ebene führen kann. Tierversuche lassen vermuten, dass für das unreife $\mathrm{Ge}$ hirn während der Phase des größten Hirnwachstums (von der Mitte der Schwanger- schaft bis zum 3. Lebensjahr) das Risiko für eine Schädigung am größten ist [40]. Dies betrifft u. a. schädigende Einflüsse auf die glialen Progenitorzellen und die Myelinisierung [41].

Andere Veröffentlichungen gehen von der Annahme aus, dass die Verwendung von Raumluft im Gegensatz zu höher konzentriertem Sauerstoff während der Erstversorgung $\mathrm{zu}$ einem verzögerten Abfall des pulmonalen Gefäßwiderstands führt. Zwei Studien haben demonstriert, dass der pulmonale Gefäßwiderstand tatsächlich bei Verwendung von höher konzentriertem Sauerstoff etwas tiefer und etwas schneller fällt. Allerdings bedeutet dies eine Erhöhung der Konzentration reaktiver Sauerstoffverbindungen, die im weiteren Verlauf einen ungünstigen Einfluss auf die pulmonal-arterielle Gefäßrelaxation haben.

Zahlreiche Studien beschäftigen sich mit der Messung der peripheren Sättigung durch Pulsoxymetrie nach der Geburt. Mit den seit Anfang des neuen Jahrtausends verfügbaren Messgeräten lassen sich innerhalb von 2 min nach der Geburt bei über 90\% der gesunden Neugeborenen, nahezu 80\% der Frühgeborenen und 80-90\% der Neugeborenen, die offenbar eine Reanimation benötigten, verlässliche Sättigungswerte messen [42]. Unbeeinträchtigte reife Neugeborene haben unter der Geburt eine arterielle Sauerstoffsättigung $\left(\mathrm{S}_{\mathrm{a}} \mathrm{O}_{2}\right)$ von etwa $60 \%$ (gemessen auf Höhe Normalnull; [43]). Diese steigt innerhalb von $10 \mathrm{~min}$ auf Werte über 90\% an [44]. Die 25. Perzentile der postnatalen $\mathrm{S}_{\mathrm{a}} \mathrm{O}_{2}$-Werte liegt etwa bei $40 \%$ bei der Geburt und steigt auf etwa $80 \%$ in der 10. Lebensminute ([45]; • Abb. 6). Bei Kindern, die durch Kaiserschnitt [46] und in größerer Höhe [47] geboren werden, sind diese Werte noch niedriger. Frühgeborene benötigen teilweise länger um $\mathrm{S}_{\mathrm{a}} \mathrm{O}_{2}$ Werte über $90 \% \mathrm{zu}$ erreichen [45]. Neugeborene, denen unterstützend Sauerstoff gegeben wurde, hatten trotz Protokoll zur Verringerung der inspiratorischen Sauerstoffkonzentration $\left(\mathrm{F}_{\mathrm{I}} \mathrm{O}_{2}\right)$ häufiger Sättigungswerte über 95\%. Diese Studien sind allerdings aufgrund zu geringer Aussagekraft und des Studiendesigns nur eingeschränkt beurteilbar [48, 49].

Empfehlung. Bei der Reanimation eines reifen Neugeborenen ist es am besten, die Beatmungen zunächst mit Raumluft statt mit 100\%igem Sauerstoff zu beginnen. Kommt es trotz suffizienter Ventilationen zu keinem zufriedenstellenden Anstieg der Herzfrequenz oder peripheren Sättigung (wenn immer möglich gemessen durch Pulsoxymetrie), wird die zugeführte Sauerstoffkonzentration erhöht.

Da viele Frühgeborene vor der 32. SSW die erwünschten peripheren Sättigungswerte häufig unter Raumluft nicht erreichen, soll für ihre Versorgung eine idealerweise durch Pulsoxymetrie überwachte zielgerichtete Sauerstoffgabe mithilfe eines Sauerstoff-Raumluft-Mischers erfolgen. Sowohl eine Hyperoxämie als auch eine Hypoxämie sind zu vermeiden. Steht kein Sauerstoff-Raumluft-Mischer zur Verfügung, soll die Reanimation mit Raumluft begonnen werden.

\section{Abnabelungszeitpunkt}

Cineradiographische Studien Neugeborener während der ersten Atemzüge nach der Geburt haben gezeigt, dass es bei Neugeborenen, deren Nabelschnur vor dem ersten Atemzug geklemmt wurde, zu einer sofortigen Verminderung der Herzgröße für die folgenden 3-4 Herzzyklen kommt. Danach zeigte das Herz wieder eine Größenzunahme etwa entsprechend der Größe des fetalen Herzens. Die Größenabnahme lässt sich durch die Füllung des neu durchbluteten pulmonalen Gefäßsystems nach Öffnung der Lungen erklären. Die in weiterer Folge zu beobachtende Größenzunahme lässt sich durch das von den Lungen zum Herzen zurückfließende Blut interpretieren [50]. Brady u. James [51] beschrieben eine Bradykardie nach Klemmen der Nabelschnur vor dem ersten Atemzug, die sich bei Klemmen nach Etablierung der Atmung nicht nachweisen ließ. Es stellt sich die Frage, ob ein zu frühes Klemmen der Nabelschnur bei einem Frühgeborenen, dessen Fähigkeit der Lungeneröffnung durch das Generieren negativer intrathorakaler Drücke begrenzt ist, eine Bradykardie auslösen oder verlängern kann und dies zu einer höheren Wahrscheinlichkeit für die Notwendigkeit von Reanimationsmaßnahmen führt.

Studien reifer Neugeborener haben bei spätem Abnabeln eine Verbesserung des Eisenstatus und weiterer Werte des ro- 
ten Blutbilds in den ersten 3 bis 6 Monaten postnatal gezeigt. Allerdings haben die spät abgenabelten Kinder auch häufiger eine Fototherapie im Rahmen einer Hyperbilirubinämie benötigt. Wobei die Durchführung der Fototherapie in diesen Studien weder genau kontrolliert noch definiert war, und diesem Umstand eher geringere klinische Bedeutung zugemessen wird.

Studien in der Population der Frühgeborenen haben (bei spätem Abnabeln) überwiegend eine verbesserte klinische Stabilisierung in der direkten postnatalen Phase sowie einen geringeren Bedarf an Bluttransfusionen in den folgenden Wochen gezeigt. Einige Studien scheinen für eine geringere Inzidenz an intraventrikulären Blutungen und „Late-onset“-Septitiden zu sprechen [52]. Auch hier berichten einige Studien vom häufigeren Auftreten einer Hyperbilirubinämie und der vermehrten Notwendigkeit einer Fototherapie. Eine erhöhte Inzidenz von Austauschtransfusionen ist allerdings nicht beschrieben.

Es gibt bisher keine Daten, die den Einfluss eines späteren Abnabelns bei Neugeborenen, die Reanimationsmaßnahmen benötigen, beschreiben, da diese Kinder aus den Studien ausgeschlossen wurden.

Empfehlung. Unbeeinträchtigte Neugeborene, die keine Reanimationsmaßnahmen benötigen, sollen verzögert, nach frühestens 1 min, abgenabelt werden. Dies soll auch für stabile Frühgeborene gelten. Für Neugeborene, die Reanimationsmaßnahmen benötigen, hat der unverzögerte Beginn von Reanimationsmaßnahmen weiter oberste Priorität.

\section{Initiale Beatmungshübe und assistierte Beatmung}

Beim reifen Neugeborenen wird durch eine suffiziente Spontanatmung oder durch assistierte initiale Beatmungen eine funktionelle Residualkapazität hergestellt („functional residual capacity“ FRC; [53, $54,55,56,57,58,59])$. Die optimalen Werte für Beatmungsdruck, Inspirationszeit und Gasfluss, die zur Etablierung einer effektiven FRC notwendig sind, sind unbekannt. Bei einem nichtspontanatmenden, reifen Neugeborenen führen Beatmungen mit einem durchschnittlichen Spitzendruck von $30-40 \mathrm{CmH}_{2} \mathrm{O}$ zu einer suffi-

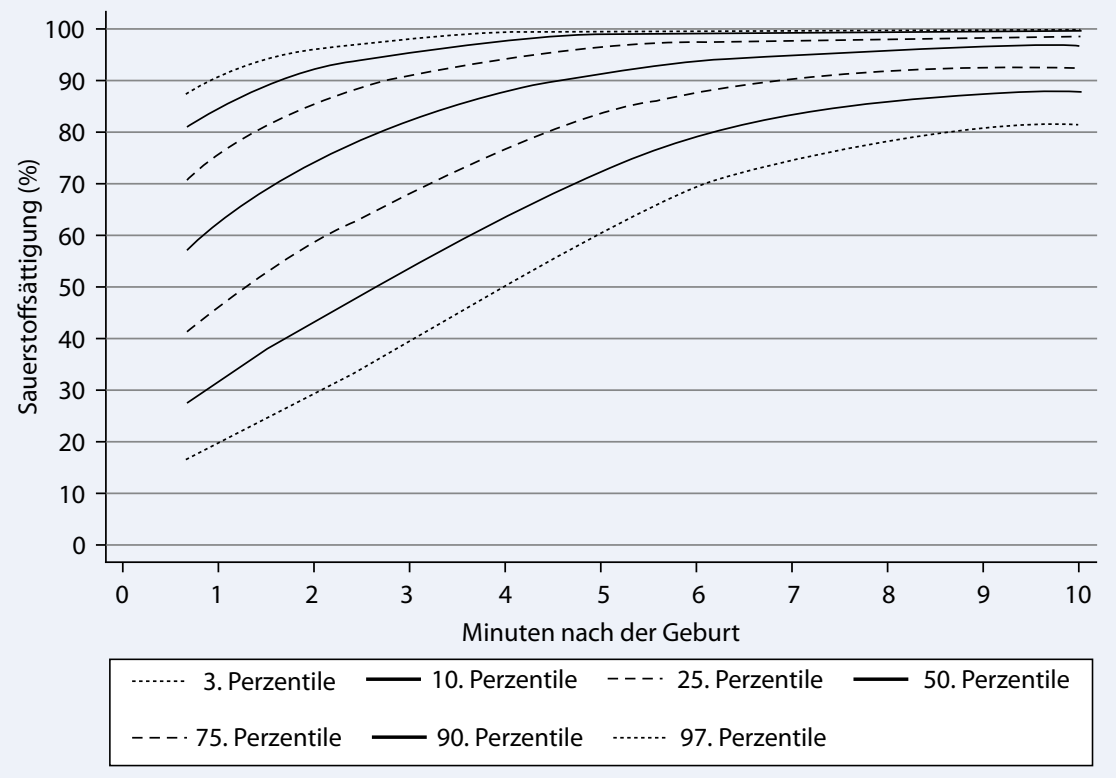

Abb. $6 \Delta$ Periphere Sauerstoffsättigung bei gesunden Neugeborenen ohne medizinische Intervention. (Mit freundlicher Genehmigung von Dawson et al. [45])

zienten Ventilation (bei nichtdefinierter Inspirationsdauer; $[54,56,57,59])$. Üblicherweise beträgt die Frequenz 30-6o Beatmungen/min. Untersuchungen über die Effektivität unterschiedlicher Beatmungsfrequenzen gibt es bisher jedoch nicht.

Misst man während der initialen Beatmungen den Beatmungsdruck, zeigt sich, dass ein Wert von $20 \mathrm{cmH}_{2} \mathrm{O}$ zum Öffnen der Lungen meist ausreichend ist. Bei Reifgeborenen sind jedoch teilweise Spitzendrücke von $30-40 \mathrm{cmH}_{2} \mathrm{O}$ und höher für die ersten Beatmungen notwendig. Wird der Beatmungsdruck nicht gemessen, sondern lediglich durch ein nichtverstellbares Überdruckventil begrenzt, soll der geringstmögliche Beatmungsdruck appliziert werden, der zu einem Anstieg der Herzfrequenz führt. Es gibt derzeit keine ausreichende Evidenz zur Empfehlung einer optimalen Inspirationszeit.

Zusammengefasst: Versuchen Sie 306o Beatmungen/min durchzuführen, um einen raschen Anstieg der Herzfrequenz über 10o/min zu erreichen bzw. die Herzfrequenz über 100/min zu halten.

\section{Assistierte Beatmung von Frühgeborenen}

Im Tiermodell zeigt sich, dass bei Frühgeborenen eine Beatmung mit hohen Tidalvolumina direkt nach der Geburt bereits zu einer Schädigung der Lungen führen kann, das Aufrechterhalten eines po- sitiven endexspiratorischen Drucks („positive end-expiratory pressure“, PEEP) direkt nach der Geburt dagegen vor einer Lungenschädigung schützt. Eine PEEPApplikation verbessert zudem die Lungen-Compliance und erleichtert den Gasaustausch [61, 62].

In Tierversuchen führte sowohl eine Überdehnung der Alveolen als auch der wiederholte Kollaps der zuvor geöffneten Alveolen zu einer Schädigung des Lungengewebes. Durch die alleinige Messung des Beatmungsdrucks kann keine ausreichende Begrenzung des Tidalvolumens erreicht werden. Um eine Überdehnung der Alveolen zu vermeiden, soll idealerweise das Tidalvolumen direkt gemessen und nach den initialen Beatmungen zum Öffnen der Lungen auf $4-8 \mathrm{ml} / \mathrm{kgKG}$ begrenzt werden [63].

Während der Beatmung von Frühgeborenen können sehr deutliche passive Thoraxbewegungen bereits Anzeichen für zu hohe Tidalvolumina sein und sollen vermieden werden. Die Anzeige des Beatmungsdrucks während der Beatmung kann helfen, eine gleichmäßigere Belüftung zu erleichtern und überhöhte Spitzendrücke zu vermeiden. Ist die Beatmung notwendig, liegt der initiale Beatmungsdruck zum Öffnen der Lungen für die meisten Frühgeborenen etwa bei 20$25 \mathrm{cmH}_{2} \mathrm{O}[64,65]$. Höhere Beatmungsdrücke sind möglicherweise notwendig, wenn es nicht zu einem raschen Anstieg 
der Herzfrequenz kommt oder keine Thoraxexkursionen feststellbar sind. Ist eine längere Beatmung notwendig, kann eine PEEP-Applikation vorteilhaft sein.

Die Verwendung eines ,continuous positive airway pressure“ (CPAP) kann beim spontan atmenden Frühgeborenen im weiteren Verlauf ebenfalls sinnvoll sein [65].

\section{Hilfsmittel zur Beatmung}

Sowohl mit einem Beutel, dessen Füllung abhängig vom Gasfluss ist (Anästhesiebeutel; Anmerkung der Übersetzer) oder einem sich selbst füllenden Beatmungsbeutel und auch mit einem mechanischen Modell mit T-Stück-System zur Regulierung des applizierten Spitzendrucks können effektive Beatmungen durchgeführt werden $[66,67,68]$. Dabei ist zu beachten, dass sich das Überdruckventil selbstfüllender Beatmungsbeutel in Abhängigkeit von der Geschwindigkeit des darüberfließenden Gases öffnet. Unter starker Beatmung können die applizierten Beatmungsdrücke daher durchaus den vom Hersteller angegebenen Spitzenöffnungsdruck übersteigen [69]. Die Verwendung eines Systems mit T-Stück erlaubt im Gegensatz zu herkömmlichen Beatmungsbeuteln eine bessere Kontrolle des applizierten Spitzendrucks und verlängerte Inspirationszeiten [70], auch wenn deren klinische Bedeutung derzeit nicht eindeutig ist. Die Verwendung eines anästhesiologischen Beutels (Füllung abhängig vom Gasfluss) erfordert mehr Übung, um ausreichende Beatmungsdrücke zu erreichen, als die Verwendung eines selbstfüllenden Beatmungsbeutels [71].

Sowohl Anästhesiebeutel, sich selbst füllende Beatmungsbeutel als auch mechanische T-Stück-Modelle sind konstruiert, um den Beatmungsdruck zu regulieren sowie zu begrenzen und sind zur Beatmung von Neugeborenen geeignet.

\section{Larynxmasken}

Zahlreiche Studien haben eine effektive Ventilation von Neugeborenen $>\mathbf{2 0 0 0}$ g, jenseits der 33. SSW und unter Reanimationsbedingungen mithilfe der Larynxmaske (LMA) gezeigt. Fallberichte beschreiben die erfolgreiche Verwendung von LMA nach erfolglosen Intubationsversuchen, teilweise war aber auch nach erfolgloser Platzierung einer LMA die Intubation notwendig. Es gibt wenige Daten bezüglich der Verwendung von LMA bei kleineren oder weniger reifen Neugeborenen.

Empfehlung. Larynxmasken können im Rahmen der Reanimation von Neugeborenen verwendet werden, besonders wenn eine Maskenbeatmung insuffizient oder eine endotracheale Intubation nicht erfolgreich oder nicht möglich ist. Eine LMA kann als Alternative zur Maskenbeatmung bei Neugeborenen $>2000$ g und $\geq 34$ SSW in Erwägung gezogen werden. Allerdings gibt es wenig Evidenz für die Verwendung von LMA bei Neugeborenen <2000 $g$ und $<34$ SSW. Eine LMA kann ebenfalls als Alternative zweiter Wahl zur endotrachealen Intubation während der Reanimation von Neugeborenen $>2000 \mathrm{~g}$ und $\geq 34$ SSW in Erwägung gezogen werden $[72,73,74]$. Die Verwendung von LMA im Rahmen von Geburten mit mekoniumhaltigem Fruchtwasser, unter Herzdruckmassagen oder zur Gabe von endotrachealen Notfallmedikamenten wurde bisher nicht untersucht.

\section{Kohlendioxidmessung während der Beatmung mit Maske oder Larynxmaske}

Detektoren zur kolorimetrischen Messung von $\mathrm{CO}_{2}$ unter Maskenbeatmung wurden bisher bei wenigen Frühgeborenen auf der Intensivstation [75] und im Kreißsaal eingesetzt [76]. Möglicherweise ist die Verwendung dieser Detektoren hilfreich, um eine Atemwegsobstruktion zu erkennen. Allerdings konnte bisher kein zusätzlicher Vorteil zur alleinigen klinischen Beurteilung eines offenen Atemwegs gezeigt werden. Auch mögliche Risiken wurden bisher nicht untersucht. Über die Verwendung von $\mathrm{CO}_{2}$-Detektoren im Zusammenhang mit anderen Atemwegshilfsmitteln (z. B. Wendel-Tubus, LMA) während der Beatmung im Kreißsaal gibt es bisher ebenfalls keine Untersuchungen.

\section{Überprüfung der endotrachealen Tubuslage}

Eine endotracheale Intubation kann während einer Neugeborenenreanimation erwogen werden:

- wenn ein Absaugen von Mekonium oder anderer die Atemwege verlegende Substanzen erforderlich ist,
- wenn eine Maskenbeatmung nicht effektiv ist oder länger durchgeführt werden muss,

- wenn Herzdruckmassagen durchgeführt werden müssen oder

- in speziellen Situationen (z. B. kongenitale Zwerchfellhernie oder Frühgeborene $<1000 \mathrm{~g}$ )

Ob und wann ein Neugeborenes intubiert wird, richtet sich nach den Fähigkeiten und den Erfahrungen der Versorgenden. Die Tubustiefe in Abhängigkeit von der Gestationswoche ist in $\bullet$ Tab. 1 aufgeführt.

Die korrekte Tubuslage muss während der Intubation unter direkter Sicht gesichert und die Tubustiefe überprüft werden. Ein prompter Anstieg der Herzfrequenz unter Beatmung ist ein guter Indikator für eine Tubuslage im tracheobronchialen System [78]. Der Nachweis von $\mathrm{CO}_{2}$ in der Ausatemluft ist eine effektive Methode zum Nachweis der Tubuslage unterhalb des Larynx bei Neugeborenen und sehr kleinen Frühgeborenen (,very low birth weight infants", VLBW; [79, 80, 81, 82]). Mehrere Neonatalstudien belegen, dass der Nachweis von ausgeatmetem $\mathrm{CO}_{2}$ bei vorliegendem Spontankreislauf schneller und zuverlässiger eine endotracheale Intubation anzeigt, als es durch klinische Beurteilung allein möglich ist $[81,82,83]$. Lässt sich kein $\mathrm{CO}_{2}$ nachweisen, macht dies eine ösophageale Tubuslage sehr wahrscheinlich, allerdings wurden im Atem-Kreislauf-Stillstand [79] und bei VLBW falsch-negative Ergebnisse trotz scheinbarer Effektivität der Methode berichtet [84].

Neugeborene, die intensive Reanimationsmaßnahmen benötigten, wurden aus den Studien ausgeschlossen. Bisher gibt es nicht genug vergleichende Untersuchungen, um eine bestimmte Methode der $\mathrm{CO}_{2}$-Erfassung für die Gruppe der Neugeborenen zu empfehlen. Falsch-positive Ergebnisse sind bei der Verwendung von Detektoren, die $\mathrm{CO}_{2}$ durch einen Farbumschlag anzeigen, möglich, wenn diese mit Adrenalin, Surfactant oder Atropin in Kontakt kommen [75].

Eine geringe oder fehlende pulmonale Perfusion oder eine Obstruktion der Trachea kann trotz korrekter endotrachealer Tubuslage einen Nachweis von ausgeatmetem $\mathrm{CO}_{2}$ verhindern. Bei fast allen $\mathrm{Pa}$ - 
tienten, die sich nicht im Atem-KreislaufStillstand befinden, kann eine endotracheale Tubuslage durch den Nachweis von exspiratorischem $\mathrm{CO}_{2}$ korrekt bestimmt werden [8o]. Bei einem kritisch kranken Neugeborenen mit reduziertem Herzminutenvolumen kann der fehlende $\mathrm{CO}_{2}$-Nachweis u. U. zu einer unnötigen Extubation führen. Weitere klinische Zeichen einer korrekten Tubuslage sind das Beschlagen des Tubuslumens während der Exspiration sowie das Heben und Senken des Brustkorbs während der Beatmung. Allerdings wurden diese Methoden bisher nicht systematisch bei Neugeborenen untersucht.

Empfehlung. Bei Neugeborenen mit Spontankreislauf ist der Nachweis von $\mathrm{CO}_{2}$ in der Ausatemluft in Ergänzung zur klinischen Beurteilung die zuverlässigste Methode, um eine endotracheale Tubuslage zu bestimmen.

\section{Verabreichungsweg und Dosis von Adrenalin}

Trotz der weit verbreiteten Verwendung von Adrenalin während der Reanimation gibt es keine placebokontrollierten Humanstudien, die die Effektivität, die optimale Dosis oder den idealen Verabreichungsweg von Adrenalin untersucht hätten.

Neonatale Fallserien und Fallberichte $[85,86]$ weisen darauf hin, dass durch eine endotracheale Gabe von Adrenalin eine Wiederherstellung eines Spontankreislaufs („return of spontaneous circulation“, ROSC) bzw. ein Anstieg der Herzfrequenz erreicht werden kann. Die verwendeten Dosen für die endotrachealen Gaben reichten in diesen Studien von 3$250 \mu \mathrm{g} / \mathrm{kgKG}$. Aufgrund des Studiendesigns (nichtstandardisierte Adrenalingabe, „bias“ in der Auswahl und Erfassung) ist die Beurteilbarkeit dieser Fallstudien allerdings eingeschränkt.

Eine Fallstudie von guter Qualität beurteilt die endotracheale Gabe von Adrenalin als weniger effektiv als eine i.v.-Gabe der gleichen Dosis (10 $\mu \mathrm{g} / \mathrm{kgKG}$; [87]). Diese Beobachtung deckt sich mit extrapolierten Daten aus Untersuchungen an neonatalen Tiermodellen. Hier waren endotracheal höhere Adrenalindosen von 50-100 $\mu \mathrm{g} / \mathrm{kgKG}$ notwendig, um die gleiche Adrenalinkonzentration im Blut und die gleiche hämodynamische Wirksamkeit wie nach i.v.-Gabe zu erzielen $[88,89]$. Untersuchungen an adulten Tiermodellen zeigten eine signifikant niedrigere Adrenalinkonzentration im Blut nach endotrachealer Gabe im Vergleich zur i.v.-Gabe [90, 91]. Um einen ROSC zu erzielen, sind möglicherweise endotracheale Dosen von 50-100 $\mu \mathrm{g} / \mathrm{kgKG}$ erforderlich [92].

Bisher gibt es keine klinischen Studien, die die weit verbreitete Annahme belegen, dass die endotracheale Adrenalingabe schneller erfolgen kann als die i.v.-Gabe. In 2 Arbeiten wurde eine inadäquat frühe endotracheale Adrenalingabe vor Öffnung des Atemwegs und der Lungen beschrieben $[85,86]$. Eine Fallstudie, die innerklinische kindliche Atem-Kreislauf-Stillstände untersuchte, konnte eine bessere Überlebenswahrscheinlichkeit für die Kinder nachweisen, die die erste Adrenalingabe endotracheal erhielten. Allerdings finden sich keine Angaben über den Zeitpunkt der endotrachealen im Vergleich zur i.v.-Gabe [93]. Hohen Adrenalindosen (100 $\mu \mathrm{g} / \mathrm{kgKG})$ unter Reanimation konnten in pädiatrischen Studien $[94,95]$ und neonatalen Tiermodellen [96] keinen Vorteil, sondern im Gegenteil einen Trend zu einer erhöhten Mortalität und zu einem schlechteren neurologischen Outcome zeigen. Diese Ergebnisse stehen im Widerspruch zu einer einzelnen pädiatrischen Fallstudie, die im Vergleich zu einer historischen Kontrollgruppe eine nachweisbar höhere Wahrscheinlichkeit für einen ROSC nach hoher i.v.-Adrenalin-Dosis $(100 \mu \mathrm{g} / \mathrm{kgKG})$ beschreibt. Allerdings konnte eine Metaanalyse von 5 Erwachsenenstudien trotz einer Verbesserung des ROSC nach hochdosierter Adrenalingabe keine Erhöhung der Überlebenswahrscheinlichkeit nachweisen [97].

Empfehlung. Wird Adrenalin verabreicht, soll es i.v. als Bolus in einer Dosis von 10$30 \mu \mathrm{g} / \mathrm{kgKG}$ verabreicht werden. Höhere Konzentrationen können schaden und sollen nicht gegeben werden. Ist kein i.v.Zugang verfügbar, kann die endotracheale Gabe sinnvoll sein. Wird Adrenalin endotracheal verabreicht, sind wahrscheinlich höhere Dosen (50-100 $\mu \mathrm{g} / \mathrm{kgKG}$ ) notwendig, um den gleichen Effekt wie bei i.v.-Gabe zu erzielen.
Tab. 1 Tubustiefe bei oraler Intubation im Verhältnis zur Gestationswoche

\begin{tabular}{ll} 
Gestationswoche & $\begin{array}{l}\text { Tubustiefe ab } \\
\text { Unterlippe (in cm) }\end{array}$ \\
\hline $23-24$ & 5,5 \\
$25-26$ & 6,0 \\
$27-29$ & 6,5 \\
\hline $30-32$ & 7,0 \\
\hline $33-34$ & 7,5 \\
$35-37$ & 8,0 \\
$38-40$ & 8,5 \\
$41-43$ & 9,0 \\
\hline
\end{tabular}

\section{Weitere Versorgung nach der Reanimation}

Auch nach anfänglicher Stabilisierung können sich Neugeborene nach einer Reanimation im weiteren Verlauf erneut klinisch verschlechtern. Sobald Beatmung und Herz-Kreislauf-System stabilisiert sind, muss das Neugeborene an einem Ort verbleiben, an dem eine engmaschige Überwachung und eine weitere Behandlung erfolgen kann, oder es muss an einen solchen Ort verlegt werden.

\section{Glucose}

In einem tierexperimentellen Asphyxieund Reanimationsmodell ließ sich eine Hypoglykämie mit einem schlechten neurologischen Outcome assoziieren [98]. Neugeborene Tiere, die während eines anoxischen oder hypoxisch-ischämischen Insults hypoglykämisch waren, hatten im Verlauf größere zerebrale Infarktareale und/oder eine geringere Überlebensrate als die Kontrollgruppe [99, 100]. Eine klinische Studie konnte nachweisen, dass eine Hypoglykämie im Rahmen einer perinatalen Asphyxie mit einem schlechten neurologischen Outcome verbunden ist [101]. Bei Erwachsenen, Kindern und extrem unreifen Neugeborenen, die intensivmedizinisch betreut werden, ist dagegen eine Hyperglykämie ebenfalls mit einem schlechten Outcome verbunden $[102,103,104]$. Eine Hyperglykämie speziell nach einem hypoxisch-ischämischen Ereignis bei pädiatrischen Patienten scheint allerdings nicht schädlich zu sein [105]. Dies unterstützen Erkenntnisse aus Studien an Tiermodellen [106], wobei manche dieser Studien sogar auf einen protektiven Effekt einer Hyperglykämie hinweisen [107]. Die verfügbare Datenlage er- 
möglicht keine Festlegung eines Blutglucosebereichs, der mit einer geringstmöglichen Hirnschädigung nach Asphyxie und Reanimation verbunden ist. Bei Neugeborenen, die reanimiert wurden, sollen die Blutglucosespiegel überwacht und im Normbereich gehalten werden.

\section{Therapeutische Hypothermie}

Mehrere randomisierte kontrollierte Multizenterstudien konnten nachweisen, dass die Mortalität bei Neugeborenen nach der 36. SSW mit moderater bis schwerer hypoxisch-ischämischen Enzephalopathie durch eine therapeutische Hypothermie $\left(33,5-34,5^{\circ} \mathrm{C}\right)$ signifikant gesenkt und ein besseres neurologischen Outcome mit 18 Monaten erzielt werden konnte [108, 109, 110, 111]. Die Gesamtkörperkühlung und die selektive Kopfkühlung führten $\mathrm{zu}$ vergleichbaren Ergebnissen [109, 110, 111, 112, 113]. Eine moderate Hypothermie führt i. Allg. zu einer Verlangsamung der Herzfrequenz und zu einem erhöhten Blutdruck; dies erfordert nicht notwendigerweise eine therapeutische Intervention. Eine rasche Erwärmung jedoch kann $\mathrm{zu}$ niedrigen Blutdrücken führen [114].

Eine tiefe Hypothermie $\left(<33^{\circ} \mathrm{C}\right.$-Kerntemperatur) kann in Arrhythmien, Blutungen, Thrombosen und Sepsis münden. Keine dieser Komplikationen ist bisher bei Neugeborenen unter moderater therapeutischer Hypothermie beschrieben worden $[109,115]$.

Reifgeborene und nahezu reife Neugeborene mit moderater bis schwerer hypoxisch-ischämischer Enzephalopathie sollen, wenn möglich, einer therapeutischen Hypothermie zugeführt werden. Sowohl die komplette Körperkühlung als auch die selektive Kopfkühlung sind geeignete Methoden. Für den Beginn und die Durchführung der Kühlung sollen standardisierte Protokolle vorliegen. Die Therapie soll auf einer neonatologischen Intensivstation in Zusammenarbeit mit einem multidisziplinären Team erfolgen. Die Behandlungsprotokolle sollen den in den randomisierten Studien verwendeten Studienprotokollen entsprechen (u. a. Beginn innerhalb von $6 \mathrm{~h}$ postnatal, Kühlungsdauer $72 \mathrm{~h}$, Wiedererwärmung über mindestens $4 \mathrm{~h}$ ). Tiermodelle zeigen deutlich, dass die Effektivität der Kühlung durch einen frühzeitigen Beginn günstig beeinflusst wird. Es gibt bei
Neugeborenen keinen Hinweis, dass eine Kühlung, die erst nach $6 \mathrm{~h}$ postnatal begonnen wird, noch effektiv ist. Bekannte Nebenwirkungen einer therapeutischen Kühlung, wie Thrombozytopenie und Hypotension, müssen engmaschig überwacht werden. Alle mit Hypothermie behandelten Neugeborenen sollen eine Nachsorge erhalten.

\section{Verzicht und Abbruch einer Reanimation}

Mortalität und Morbidität von Neugeborenen variieren regional und sind von der Verfügbarkeit medizinischer Ressourcen abhängig [116]. Sozialwissenschaftliche Studien belegen, dass Eltern schwer kranker Neugeborener stärker in Entscheidungsprozesse bezüglich Reanimationsmaßnahmen und weitergehenden lebensverlängernden Maßnahmen eingebunden werden wollen [117]. Die Vor- und Nachteile einer aggressiven Intensivtherapie bei schwer kranken Neugeborenen werden von medizinisch Handelnden, Eltern und in der Gesellschaft sehr unterschiedlich beurteilt $[118,119]$.

\section{Verzicht auf Reanimationsmaßnahmen}

Es gibt Umstände, in denen die Prognose eines Neugeborenen mit einer hohen Mortalität und einem schlechten Outcome verbunden ist. Hier kann es statthaft sein, keine Reanimation zu beginnen, insbesondere dann, wenn die Gelegenheit zur vorherigen Besprechung mit den Eltern bestand [24, 120, 121].

Jeder Fall muss vom geburtshilflichen und neonatologischen Team als individuell betrachtet werden, und Entscheidungen sollen in übereinstimmender und koordinierter Vorgehensweise gemeinsam mit den Eltern getroffen werden [23]. Eine Reanimation nicht zu beginnen oder lebenserhaltende Maßnahmen während oder nach einer Reanimation zu beenden, wird i. Allg. als ethisch gleichwertige Entscheidungen angesehen. Daher soll nicht gezögert werden, lebenserhaltende Maßnahmen auch im Verlauf beenden, wenn ein Überleben mit schwersten funktionellen Störungen höchstwahrscheinlich ist.

Die folgenden Leitlinien müssen vor dem Hintergrund regional unterschied- licher Daten zum Outcome interpretiert werden:

- Wenn Gestationsalter, Geburtsgewicht und/oder kongenitale Fehlbildungen mit an Sicherheit grenzender Wahrscheinlichkeit mit einem frühen Tod verbunden sind und die sehr wenigen Überlebenden eine inakzeptabel hohe Morbidität aufweisen, ist eine Reanimation nicht angezeigt [122]. Beispiele aus der publizierten Literatur beinhalten Frühgeburtlichkeit mit einem Gestationsalter $<23$ SSW und/oder einem Geburtsgewicht $<400$ g sowie Anomalien wie Anenzephalie oder eine nachgewiesene Trisomie 13 oder 18 .

- Der Beginn von Reanimationsmaßnahmen ist fast immer angezeigt, wenn eine hohe Überlebensrate und eine vertretbare Morbidität wahrscheinlich sind. Darunter fallen grundsätzlich Frühgeborene ab der 25. Gestationswoche (es sei denn, es gibt Hinweise auf schwer wiegende fetale Probleme wie eine schwere intrauterine Infektion oder eine Hypoxie bzw. Ischämie) sowie die Mehrzahl der kongenitalen Fehlbildungen.

- Ist die Prognose für ein Kind aufgrund unklarer Überlebensrate mit relativ hoher Morbidität unsicher und sind die für das Kind anzunehmenden Belastungen hoch, sollen die Wünsche der Eltern bezüglich zu treffender Maßnahmen unterstützt werden.

\section{Beendigung von Reanimationsmaßnahmen}

Neugeborene, die vom Zeitpunkt der Geburt an für mindestens 10 min oder länger keine Lebenszeichen aufweisen, haben eine hohe Mortalität oder schwerste entwicklungsneurologische Beeinträchtigungen $[123,124]$. Es ist daher statthaft, eine Beendigung der Reanimationsmaßnahmen zu erwägen, wenn ein Neugeborenes nach 10-minütigen ununterbrochenen und adäquaten Reanimationsbemühungen keine Lebenszeichen zeigt. Die Entscheidung, eine Reanimation nach mehr als 10 min ohne Herzaktion weiterzuführen, ist oft komplex und wird durch die weiteren Umstände bestimmt. Es fließt eine Vielzahl von Variablen wie die Ätiologie des Atem-Kreislauf-Stillstands, das Gestationsalter, die Aussicht auf Reversi- 
bilität des Atem-Kreislauf-Stillstands und evtl. von den Eltern geäußerte Wünsche sowie Ansichten über eine vertretbare Morbidität des Kindes ein.

Liegt die Herzfrequenz nach der Geburt unter 60 Schlägen/min und bleibt sie über 10-15 min darunter, ist die Entscheidung zur Beendigung oder Fortführung von Reanimationsmaßnahmen noch deutlich schwieriger. Derzeit können keine klaren Empfehlungen für ein weiteres Vorgehen in dieser Situation ausgesprochen werden.

\section{Korrespondierende Übersetzer \\ Dr. Jens-C. Schwindt}

Universitätsklinik für Kinder- und

Jugendheilkunde

Medizinische Universität Wien

Währinger Gürtel 18-20, 1090 Wien

jens.schwindt@meduniwien.ac.at

\section{Dr. Ulrich Kreth}

Kinderkrankenhaus St. Nikolaus

AKH Viersen

Hoserkirchweg 63, 41747 Viersen

kreth@akh-viersen.de

Interessenkonflikt. Die korrespondierenden Übersetzer geben an, dass kein Interessenkonflikt besteht.

\section{Literatur}

1. Wyllie J, Perlman JM, Kattwinkel J et al (2010) International Consensus on Cardiopulmonary Resuscitation and Emergency Cardiovascular Care Science with Treatment Recommendations. Part 11: Neonatal Resuscitation. Resuscitation In Press

2. Perlman JM, Wyllie J, Kattwinkel J et al (2010) International Consensus on Cardiopulmonary Resuscitation and Emergency Cardiovascular Care Science with Treatment Recommendations. Part 11: Neonatal Resuscitation. Circulation In Press

3. Biarent D, Bingham R, Richmond S et al (2005) European Resuscitation Council guidelines for resuscitation 2005. Section 6. Paediatric life support. Resuscitation 67(Suppl 1):S97-S133

4. Palme-Kilander C (1992) Methods of resuscitation in low-Apgar-score newborn infants - a national survey. Acta Paediatr 81:739-744

5. Dahm LS, James LS (1972) Newborn temperature and calculated heat loss in the delivery room. Pediatrics 49:504-513

6. Stephenson J, Du J, TK O (1970) The effect if cooling on blood gas tensions in newborn infants. J Pediatr 76:848-852

7. Gandy GM, Adamsons K Jr, Cunningham N et al (1964) Thermal environment and acid-base homeostasis in human infants during the first few hours of life. J Clin Invest 43:751-758

8. Kent AL, Williams J (2008) Increasing ambient operating theatre temperature and wrapping in polyethylene improves admission temperature in premature infants. J Paediatr Child Health 44:325-331

9. Knobel RB, Wimmer JE Jr, Holbert D (2005) Heat loss prevention for preterm infants in the delivery room. J Perinatol 25:304-308

10. ApgarV (1953) A proposal for a new method of evaluation of the newborn infant. Curr Res Anesth Analg 32

11. Chamberlain G, Banks J (1974) Assessment of the Apgar score. Lancet 2:1225-1228
12. Owen CJ, Wyllie JP (2004) Determination of heart rate in the baby at birth. Resuscitation 60:213-217

13. Kamlin CO, Dawson JA, O'Donnell CP et al (2008) ACcuracy of pulse oximetry measurement of heart rate of newborn infants in the delivery room. J Pediatr 152:756-760

14. O'Donnell CP, Kamlin CO, Davis PG et al (2007) Clinical assessment of infant colour at delivery. Arch Dis Child Fetal Neonatal Ed 92:F465-F467

15. Cordero L Jr, Hon EH (1971) Neonatal bradycardia followingnasopharyngealstimulation.JPediatr78:441-447

16. Houri PK, Frank LR, Menegazzi JJ, Taylor R (1997) A randomized, controlled trial of two-thumb vs two-finger chest compression in a swine infant model of cardiac arrest [see comment]. Prehosp Emerg Care 1:65-67

17. David $R$ (1988) Closed chest cardiac massage in the newborn infant. Pediatrics 81:552-554

18. Menegazzi JJ, Auble TE, Nicklas KA et al (1993) Twothumb versus two-finger chest compression during CRP in a swine infant model of cardiac arrest. Ann Emerg Med 22:240-243

19. Thaler MM, Stobie GH (1963) An improved technique of external caridac compression in infants and young children. N Engl J Med 269:606-610

20. Meyer A, Nadkarni V, Pollock A et al (2010) Evaluation of the Neonatal Resuscitation Program's recommended chest compression depth using computerized tomography imaging. Resuscitation 81:544-548

21. Dean JM, Koehler RC, Schleien CL et al (1991) Improved blood flow during prolonged cardiopulmonary resuscitation with $30 \%$ duty cycle in infant pigs. Circulation 84:896-904

22. Wyckoff MH, Perlman JM, Laptook AR (2005) Use of volume expansion during delivery room resuscitation in near-term and term infants. Pediatrics 115:950-955

23. (o A) (o J) Nuffield Council on Bioethics. Critical care decisions in fetal and neonatal medicine: ethical issues. ISBN 1904384142006

24. Costeloe K, Hennessy E, Gibson AT et al (2000) The EPICure study: outcomes to discharge from hospital for infants born at the threshold of viability. Pediatrics 106:659-671

25. Vohra S, Frent G, Campbell V et al (1999) Effect of polyethylene occlusive skin wrapping on heat loss in very low birth weight infants at delivery: a randomized trial. J Pediatr 134:547-551

26. Lenclen R, Mazraani M, Jugie M et al (2002) Use of a polyethylene bag: a way to improve the thermal environment of the premature newborn at the delivery room. Arch Pediatr 9:238-244

27. Bjorklund L, Hellstrom-Westas L (2000) Reducing heat loss at birth in very preterm infants. J Pediatr 137:739-740

28. Vohra S, Roberts RS, Zhang B et al (2004) Heat Loss Prevention (HeLP) in the delivery room: $A$ randomized controlled trial of polyethylene occlusive skin wrapping in very preterm infants. J Pediatr 145:750-753

29. Lieberman E, Eichenwald E, Mathur G et al (2000) Intrapartum fever and unexplained seizures in term infants. Pediatrics 106:983-988

30. Grether JK, Nelson KB (1997) Maternal infection and cerebral palsy in infants of normal birth weight. JAMA 278:207-211

31. Coimbra C, Boris-Moller F, Drake M, Wieloch T (1996) Diminished neuronal damage in the rat brain by late treatment with the antipyretic drug dipyrone or cooling following cerebral ischemia. Acta Neuropathol 92:447-453

32. Dietrich WD, Alonso O, Halley M, Busto R (1996) Delayed posttraumatic brain hyperthermia worsens outcome after fluid percussion brain injury: a light and electron microscopic study in rats. Neurosurgery 38:533-541; discussion 41

33. Carson BS, Losey RW, Bowes WA Jr, Simmons MA (1976) Combined obstetric and pediatric approach to prevent meconium aspiration syndrome. Am J Obstet Gynecol 126:712-715

34. Ting P, Brady JP (1975) Tracheal suction in meconium aspiration. Am J Obstet Gynecol 122:767-771
35. Falciglia HS, Henderschott C, Potter P, Helmchen R (1992) Does DeLee suction at the perineum prevent meconium aspiration syndrome? Am J Obstet Gynecol 167:1243-1249

36. Wiswell TE, Tuggle JM, Turner BS (1990) Meconium aspiration syndrome: have we made a difference? Pediatrics 85:715-721

37. Wiswell TE, Gannon CM, Jacob J et al (2000) Delivery room management of the apparently vigorous meconium-stained neonate: results of the multicenter, international collaborative trial. Pediatrics 105:1-7

38. Vain NE, Szyld EG, Prudent LM et al (2004) Oropharyngeal and nasopharyngeal suctioning of meconium-stained neonates before delivery of their shoulders: multicentre, randomised controlled trial. Lancet 364:597-602

39. Davis PG, Tan A, O'Donnell CP, Schulze A (2004) Resuscitation of newborn infants with $100 \%$ oxygen or air:a systematic review and meta-analysis. Lancet 364:1329-1333

40. Felderhoff-Mueser U, Bittigau P, Sifringer M et al (2004) Oxygen causes cell death in the developing brain. Neurobiol Dis 17:273-282

41. Koch JD, Miles DK, Gilley JA et al (2008) Brief exposure to hyperoxia depletes the glial progenitor pool and impairs functional recovery after hypoxic-ischemic brain injury. J Cereb Blood Flow Metab 28:1294-1306

42. O'Donnell CP, Kamlin CO, Davis PG, Morley CJ (2005) Feasibility of and delay in obtaining pulse oximetry during neonatal resuscitation. J Pediatr 147:698-699

43. Dildy GA, Berg PP van den, Katz M et al (1994) Intrapartum fetal pulse oximetry: fetal oxygen saturation trends during labor and relation to delivery outcome. Am J Obstet Gynecol 171:679-684

44. Mariani G, Dik PB, Ezquer A et al (2007) Pre-ductal and post-ductal 02 saturation in healthy term neonates after birth. J Pediatr 150:418-421

45. Dawson JA, Kamlin CO, Vento M et al (2010) Defining the reference range for oxygen saturation for infants after birth. Pediatrics 125:e1340-e1347

46. Rabi Y, YeeW, Chen SY, Singhal N (2006) Oxygen saturation trends immediately after birth.J Pediatr 148:590-594

47. Gonzales GF, Salirrosas A (2005) Arterial oxygen saturation in healthy newborns delivered at term in Cerro de Pasco (4340 m) and Lima (150 m). Reprod Biol Endocrinol 3:46

48. Escrig R, Arruza L, Izquierdo l et al (2008) Achievement of targeted saturation values in extremely low gestational age neonates resuscitated with low or high oxygen concentrations: a prospective, randomized trial. Pediatrics 121:875-881

49. Wang CL, Anderson C, Leone TA et al (2008) Resuscitation of preterm neonates by using room air or $100 \%$ oxygen. Pediatrics 121:1083-1089

50. Peltonen T (1981) Placental transfusion-advantage an disadvantage. Eur J Pediatr 137:141-146

51. Brady JP, James LS (1962) Heart rate changes in the fetus and newborn infant during labor, delivery, and the immediate neonatal period. Am J Obstet Gynecol 84:1-12

52. Mercer JS, Vohr BR, McGrath MM et al (2006) Delayed cord clamping in very preterm infants reduces the incidence of intraventricular hemorrhage and late-onset sepsis: a randomized, controlled trial. Pediatrics 117:1235-1242

53. Vyas H, Milner AD, Hopkin IE, Boon AW (1981) Physiologic responses to prolonged and slow-rise inflation in the resuscitation of the asphyxiated newborn infant. J Pediatr 99:635-639

54. Mortola JP, Fisher JT, Smith JB et al (1982) Onset of respiration in infants delivered by cesarean section. J Appl Physiol 52:716-724

55. Hull D (1969) Lung expansion and ventilation during resuscitation of asphyxiated newborn infants. J Pediatr 75:47-58

56. Upton CJ, Milner AD (1991) Endotracheal resuscitation of neonates using a rebreathing bag. Arch Dis Child 66:39-42

57. Vyas H, Milner AD, Hopkins IE (1981) Intrathoracic pressure and volume changes during the spontaneous onset of respiration in babies born by cesarean section and by vaginal delivery. J Pediatr 99:787-791 
58. Vyas H, Field D, Milner AD, Hopkin IE (1986) Determinants of the first inspiratory volume and functional residual capacity at birth. Pediatr Pulmonol 2:189-193

59. Boon AW, Milner AD, Hopkin IE (1979) Lung expansion, tidal exchange, and formation of the functional residual capacity during resuscitation of asphyxiated neonates. J Pediatr 95:1031-1036

60. Ingimarsson J, Bjorklund LJ, Curstedt T et al (2004) Incomplete protection by prophylactic surfactant against the adverse effects of large lung inflations at birth in immature lambs. Intensive Care Med 30:1446-1453

61. Nilsson R, Grossmann G, Robertson B (1980) Bronchiolar epithelial lesions induced in the premature rabbit neonate by short periods of artificial ventilation. Acta Pathol Microbiol Scand 88:359-367

62. Probyn ME, Hooper SB, Dargaville PA et al (2004) Positive end expiratory pressure during resuscitation of premature lambs rapidly improves blood gases without adversely affecting arterial pressure. Pediatr Res 56:198-204

63. Schmolzer GM, Kamlin OF, Dawson JA et al (2009) Respiratory monitoring of neonatal resuscitation. Arch Dis Child Fetal Neonatal Ed

64. Hird MF, Greenough A, Gamsu HR (1991) Inflating pressures for effective resuscitation of preterm infants. Early Hum Dev 26:69-72

65. Lindner W, Vossbeck S, Hummler H, Pohlandt F (1999) Delivery room management of extremely low birth weight infants: spontaneous breathing or intubation? Pediatrics 103:961-967

66. Allwood AC, Madar RJ, Baumer JH et al (2003) Changes in resuscitation practice at birth. Arch Dis Child Fetal Neonatal Ed 88:F375-F379

67. Cole AF, Rolbin SH, Hew EM, Pynn S (1979) An improved ventilator system for delivery-room management of the newborn. Anesthesiology 51:356-358

68. Hoskyns EW, Milner AD, Hopkin IE (1987) A simple method of face mask resuscitation at birth. Arch Dis Child 62:376-378

69. Ganga-Zandzou PS, Diependaele JF, Storme L et al (1996) Is Ambu ventilation of newborn infants a simple question of finger-touch? Arch Pediatr 3:1270-1272

70. Finer NN, Rich W, Craft A, Henderson C (2001) Comparison of methods of bag and mask ventilation for neonatal resuscitation. Resuscitation 49:299-305

71. Kanter RK (1987) Evaluation of mask-bag ventilation in resuscitation of infants. Am J Dis Child 141:761-763

72. Esmail N, Saleh M et al (2002) Laryngeal mask airway versus endotracheal intubation for Apgar score improvement in neonatal resuscitation. Egypt J Anesthesiol 18:115-121

73. Trevisanuto D, Micaglio M, Pitton M et al (2004) Laryngeal mask airway: is the management of neonates requiring positive pressure ventilation at birth changing? Resuscitation 62:151-157

74. Singh R (2005) Controlled trial to evaluate the use of LMA for neonatal resuscitation. J Anaesth Clin Pharmacol 21:303-306

75. Leone TA, Lange A, Rich W, Finer NN (2006) Disposable colorimetric carbon dioxide detector use as an indicator of a patent airway during noninvasive mask ventilation. Pediatrics 118:e202-e204

76. Finer NN, Rich W, Wang C, Leone T (2009) Airway obstruction during mask ventilation of very low birth weight infants during neonatal resuscitation. Pediatrics 123:865-869

77. Kempley ST, Moreiras JW, Petrone FL (2008) Endotracheal tube length for neonatal intubation. Resuscitation 77:369-373

78. Palme-Kilander C, Tunell R (1993) Pulmonary gas exchange during facemask ventilation immediately after birth. Arch Dis Child 68:11-16

79. Aziz HF, Martin JB, Moore JJ (1999) The pediatric disposable end-tidal carbon dioxide detector role in endotracheal intubation in newborns. J Perinatol 19:110-113

80. Bhende MS, LaCovey D (1995) A note of caution about the continuous use of colorimetric end-tidal $\mathrm{CO} 2$ detectors in children. Pediatrics 95:800-801

81. Repetto JE, Donohue PCP, Baker SF et al (2001) Use of capnography in the delivery room for assessment of endotracheal tube placement. J Perinatol 21:284-287
82. Roberts WA, Maniscalco WM, Cohen AR et al (1995) The use of capnography for recognition of esophageal intubation in the neonatal intensive care unit. Pediatr Pulmonol 19:262-268

83. Hosono S, Inami I, Fujita $\mathrm{H}$ et al (2009) A role of end-tidal $\mathrm{CO}(2)$ monitoring for assessment of tracheal intubations in very low birth weight infants during neonatal resuscitation at birth. J Perinat Med 37:79-84

84. Garey DM, Ward R, Rich W et al (2008) Tidal volume threshold for colorimetric carbon dioxide detectors availableforuseinneonates.Pediatrics 121:e1524-e1527

85. Jankov RP, Asztalos EV, Skidmore MB (2000) Favourable neurological outcomes following delivery room cardiopulmonary resuscitation of infants $<0 r=750 \mathrm{~g}$ at birth. J Paediatr Child Health 36:19-22

86. O'Donnell Al, Gray PH, Rogers YM (1998) Mortality and neurodevelopmental outcome for infants receiving adrenaline in neonatal resuscitation. J Paediatr Child Health 34:551-556

87. Barber CA, Wyckoff MH (2006) Use and efficacy of endotracheal versus intravenous epinephrine during neonatal cardiopulmonary resuscitation in the delivery room. Pediatrics 118:1028-1034

88. Crespo SG, Schoffstall JM, Fuhs LR, Spivey WH (1991) Comparison of two doses of endotracheal epinephrine in a cardiac arrest model. Ann Emerg Med 20:230 234

89. Jasani MS, Nadkarni VM, Finkelstein MS et al (1994) Effects of different techniques of endotracheal epinephrine administration in pediatric porcine hypoxichypercarbic cardiopulmonary arrest. Crit Care Med 22:1174-1180

90. Mielke LL, Frank C, Lanzinger MJ et al (1998) Plasma catecholamine levels following tracheal and intravenous epinephrine administration in swine. Resuscitation 36:187-192

91. Roberts JR, Greenberg MI, Knaub MA et al (1979) Blood levels following intravenous and endotracheal epinephrine administration. JACEP 8:53-56

92. Hornchen U, Schuttler J, Stoeckel H et al (1987) Endobronchial instillation of epinephrine during cardiopulmonary resuscitation. Crit Care Med 15:1037-1039

93. Guay J, Lortie L (2004) An evaluation of pediatric inhospital advanced life support interventions using the pediatric Utstein quidelines: a review of 203 cardiorespiratory arrests. Can J Anaesth 51:373-378

94. Perondi MB, Reis AG, Paiva EF et al (2004) A comparison of high-dose and standard-dose epinephrine in children with cardiac arrest. N Engl J Med 350:17221730

95. Patterson MD, Boenning DA, Klein BL et al (2005) The use of high-dose epinephrine for patients with out-ofhospital cardiopulmonary arrest refractory to prehospital interventions. Pediatr Emerg Care 21:227-237

96. Berg RA, Otto CW, Kern KB et al (1996) A randomized, blinded trial of high-dose epinephrine versus standard-dose epinephrine in a swine model of pediatric asphyxial cardiac arrest. Crit Care Med 24:1695-1700

97. Vandycke C, Martens P (2000) High dose versus standard dose epinephrine in cardiac arrest - a meta-analysis. Resuscitation 45:161-166

98. Brambrink AM, Ichord RN, Martin し et al (1999) Poor outcome after hypoxia-ischemia in newborns is associated with physiological abnormalities during early recovery. Possible relevance to secondary brain injury after head trauma in infants. Exp Toxicol Pathol 51:151-162

99. Vannucci RC, Vannucci SJ (1978) Cerebral carbohydrate metabolism during hypoglycemia and anoxia in newborn rats. Ann Neurol 4:73-79

100. Yager JY, Heitjan DF, Towfighi J, Vannucci RC (1992) Effect of insulin-induced and fasting hypoglycemia on perinatal hypoxic-ischemic brain damage. Pediatr Res 31:138-142

101. Salhab WA, Wyckoff MH, Laptook AR, Perlman JM (2004) Initial hypoglycemia and neonatal brain injury in term infants with severe fetal acidemia. Pediatrics 114:361-366

102. Kent TA, Soukup VM, Fabian RH (2001) Heterogeneity affecting outcome from acute stroke therapy: making reperfusion worse. Stroke 32:2318-2327
103. Srinivasan V, Spinella PC, Drott HR et al (2004) Association of timing, duration, and intensity of hyperglycemia with intensive care unit mortality in critically ill children. Pediatr Crit Care Med 5:329-336

104. Hays SP, Smith EO, Sunehag AL (2006) Hyperglycemia is a risk factor for early death and morbidity in extremely low birth-weight infants. Pediatrics 118:18111818

105. Klein GW, Hojsak JM, Schmeidler J, Rapaport R (2008) Hyperglycemia and outcome in the pediatric intensive care unit. J Pediatr 153:379-384

106. LeBlanc MH, Huang M, Patel D et al (1994) Glucose given after hypoxic ischemia does not affect brain injury in piglets. Stroke 25:1443-1447; discussion 8

107. Hattori H, Wasterlain CG (1990) Posthypoxic glucose supplement reduces hypoxic-ischemic brain damage in the neonatal rat. Ann Neurol 28:122-128

108. Edwards AD, Brocklehurst P, Gunn AJ et al (2010) Neurological outcomes at 18 months of age after moderate hypothermia for perinatal hypoxic ischaemic encephalopathy: synthesis and metaanalysis of trial data. BMJ 340:c363

109. Gluckman PD, Wyatt JS, Azzopardi D et al (2005) Selective head cooling with mild systemic hypothermia after neonatal encephalopathy: multicentre randomised trial. Lancet 365:663-670

110. Shankaran S, Laptook AR, Ehrenkranz RA et al (2005) Whole-body hypothermia for neonates with hypoxic-ischemic encephalopathy. N Engl J Med 353:15741584

111. Azzopardi DV, Strohm B, Edwards AD et al (2009) Moderate hypothermia to treat perinatal asphyxial encephalopathy. N Engl J Med 361:1349-1358

112. Eicher DJ, Wagner CL, Katikaneni LP et al (2005) Moderate hypothermia in neonatal encephalopathy: efficacy outcomes. Pediatr Neurol 32:11-17

113. Lin ZL, Yu HM, Lin J et al (2006) Mild hypothermia via selective head cooling as neuroprotective therapy in term neonates with perinatal asphyxia: an experience from a single neonatal intensive care unit. J Perinatol 26:180-184

114. Thoresen M, Whitelaw A (2000) Cardiovascular changes during mild therapeutic hypothermia and rewarming in infants with hypoxic-ischemic encephalopathy. Pediatrics 106:92-99

115. Shankaran S, Laptook A, Wright LL et al (2002) Wholebody hypothermia for neonatal encephalopathy: animal observations as a basis for a randomized, controlled pilot study in term infants. Pediatrics 110:377-385

116. De Leeuw R, Cuttini M, Nadai M et al (2000) Treatment choices for extremely preterm infants: an international perspective. J Pediatr 137:608-616

117. Lee SK, Penner PL, Cox M (1991) Comparison of the attitudes of health care professionals and parents toward active treatment of very low birth weight infants. Pediatrics 88:110-114

118. Kopelman LM, Irons TG, Kopelman AE (1988) Neonatologists judge the „Baby Doe" regulations. N Engl J Med 318:677-683

119. Sanders MR, Donohue PK, Oberdorf MA et al (1995) Perceptions of the limit of viability: neonatologists' attitudes toward extremely preterm infants. J Perinatol 15:494-502

120. Draper ES, Manktelow B, Field DJ, James D (2003) Tables for predicting survival for preterm births are updated. BMJ 327:872

121. Cole TJ, Hey E, Richmond S (2010) The PREM score: a graphical tool for predicting survival in very preterm births. Arch Dis Child Fetal Neonatal Ed 95:F14-F19

122. Swamy R, Mohapatra S, Bythell M, Embleton ND (2010) Survival in infants live born at less than 24 weeks' gestation: the hidden morbidity of non-survivors. Arch Dis Child Fetal Neonatal Ed

123. Jain L, Ferre C, Vidyasagar D et al (1991) Cardiopulmonary resuscitation of apparently stillborn infants: survival and long-term outcome. J Pediatr 118:778782

124. Haddad B, Mercer BM, Livingston JC et al (2000) Outcome after successful resuscitation of babies born with apgar scores of 0 at both 1 and 5 minutes. Am J Obstet Gynecol 182:1210-1214 